\title{
Dimethyl Fumarate, an Approved Multiple Sclerosis Treatment, Reduces Brain Oxidative Stress in SIV-Infected Rhesus Macaques: Potential Therapeutic Repurposing for HIV Neuroprotection
}

\author{
Yoelvis Garcia-Mesa ${ }^{1}{ }^{\circledR}$, He N. Xu ${ }^{2}{ }^{\oplus}$, Patricia Vance ${ }^{1}$, Analise L. Gruenewald ${ }^{1}$, Rolando Garza ${ }^{1}$, Cecily Midkiff ${ }^{3}$, \\ Xavier Alvarez-Hernandez ${ }^{3}$, David J. Irwin ${ }^{1}$, Alexander J. Gill ${ }^{1}{ }^{\circledR}$ and Dennis L. Kolson ${ }^{1, * \mathbb{C}}$ \\ 1 Department of Neurology, Perelman School of Medicine, University of Pennsylvania, \\ Philadelphia, PA 19104, USA; megarcia@pennmedicine.upenn.edu (Y.G.-M.); \\ vancep2@patriciavance.com (P.V.); analise.gruenewald@gmail.com (A.L.G.); \\ garzar18@livemail.uthscsa.edu (R.G.); dirwin@pennmedicine.upenn.edu (D.J.I.); \\ agill@pennmedicine.upenn.edu (A.J.G.) \\ 2 Britton Chance Laboratory of Redox Imaging, Department of Radiology, Perelman School of Medicine, \\ University of Pennsylvania, Philadelphia, PA 19104, USA; hexu2@pennmedicine.upenn.edu \\ 3 Department of Pathology, TNPRC, Tulane University, Covington, LA 70433, USA; \\ cconerly@tulane.edu (C.M.); jalvarezhernandez@txbiomed.org (X.A.-H.) \\ check for \\ updates \\ * Correspondence: kolsond@pennmedicine.upenn.edu
}

Citation: Garcia-Mesa, Y.; Xu, H.N.; Vance, P.; Gruenewald, A.L.; Garza, R.; Midkiff, C.; Alvarez-Hernandez, X.; Irwin, D.J.; Gill, A.J.; Kolson, D.L. Dimethyl Fumarate, an Approved Multiple Sclerosis Treatment, Reduces Brain Oxidative Stress in SIV-Infected Rhesus Macaques: Potential Therapeutic Repurposing for HIV Neuroprotection. Antioxidants 2021, 10, 416. https://doi.org/ 10.3390/antiox10030416

Academic Editor: Stanley Omaye

Received: 4 February 2021

Accepted: 5 March 2021

Published: 9 March 2021

Publisher's Note: MDPI stays neutral with regard to jurisdictional claims in published maps and institutional affiliations.

Copyright: (c) 2021 by the authors. Licensee MDPI, Basel, Switzerland. This article is an open access article distributed under the terms and conditions of the Creative Commons Attribution (CC BY) license (https:/ / creativecommons.org/licenses/by/ $4.0 /)$.

\begin{abstract}
Dimethyl fumarate (DMF), an antioxidant/anti-inflammatory drug approved for the treatment of multiple sclerosis, induces antioxidant enzymes, in part through transcriptional upregulation. We hypothesized that DMF administration to simian immunodeficiency virus (SIV)-infected rhesus macaques would induce antioxidant enzyme expression and reduce oxidative injury and inflammation throughout the brain. Nine SIV-infected, CD8 ${ }^{+}$-T-lymphocyte-depleted rhesus macaques were studied. Five received oral DMF prior to the SIV infection and through to the necropsy day. Protein expression was analyzed in 11 brain regions, as well as the thymus, liver, and spleen, using Western blot and immunohistochemistry for antioxidant, inflammatory, and neuronal proteins. Additionally, oxidative stress was determined in brain sections using immunohistochemistry (8-OHdG, 3NT) and optical redox imaging of oxidized flavoproteins containing flavin adenine dinucleotide (Fp) and reduced nicotinamide adenine dinucleotide (NADH). The DMF treatment was associated with no changes in virus replication; higher expressions of the antioxidant enzymes NQO1, GPX1, and HO-1 in the brain and PRDX1 and HO-2 in the spleen; lower levels of 8-OHdG and 3NT; a lower optical redox ratio. The DMF treatment was also associated with increased expressions of cell-adhesion molecules (VCAM-1, ICAM-1) and no changes in HLA-DR, CD68, GFAP, NFL, or synaptic proteins. The concordantly increased brain antioxidant enzyme expressions and reduced oxidative stress in DMF-treated SIV-infected macaques suggest that DMF could limit oxidative stress throughout the brain through effective induction of the endogenous antioxidant response. We propose that DMF could potentially induce neuroprotective brain responses in persons living with HIV.
\end{abstract}

Keywords: SIV; HIV-associated neurocognitive disorders; fumaric acid esters; dimethyl fumarate; DMF; oxidative stress; antioxidant response; optical redox ratio; macaque; brain

\section{Introduction}

Despite the effectiveness of combination antiretroviral therapy (cART) in suppressing human immunodeficiency virus (HIV) replication in the peripheral and central nervous system (CNS) compartments, individuals remain at risk for secondary complications that are related to persistent or recurrent inflammation and oxidative stress in all compartments [1-5]. In the rhesus macaque simian immunodeficiency virus (SIV) infection model and in persons living with HIV, early and persistent damage to the brain and 
other tissues are observed [2,3,6,7]. Damage to gut-associated lymphoid tissue results in acute and chronic immune activation, inflammation, and oxidative stress. These are linked to increased microbial translocation and associated end-organ complications, particularly in the CNS $[1,2,8,9]$. The maximum prevention of such complications likely requires protective therapies that are initiated concurrently with cART that suppress these pathologic pathways.

One approach to reducing oxidative-stress-associated injury is the activation of the host endogenous antioxidant response, which is mediated in part through the activation of the nuclear-factor-E2-related factor 2 (Nrf2)/antioxidant response element (ARE) pathway. In various animal models, activation of the ARE in the brain is associated with reduced neurodegeneration, whereas suppression of the ARE may promote neurodegeneration [10]. Activation of the Nrf2/ARE pathway to regulate oxidative stress and inflammation in the brain may promote axonal regeneration and block neurotoxicity [11-15].

The fumaric acid ester family, as represented by dimethyl fumarate (DMF) and monomethyl fumarate (MMF), which is the active in vivo metabolite of DMF, can activate Nrf2/ARE signaling, as well as non-Nrf2/ARE pathways, regulating the expression of antioxidant, anti-inflammatory, and cytoprotective genes [16-19]. DMF and MMF induce the expression of Nrf2/ARE-driven genes (NAD(p)H quinone dehydrogenase 1 (NQO1), glutathione peroxidase 1 (GPX1), peroxiredoxin 1 (RDX1), and heme oxygenase-1 (HO-1), among others) when applied to cultured cells or when administered to experimental animals $[12,18,20]$. DMF and MMF can also reduce the production of nitric oxide synthase and proinflammatory cytokines IL- $1 \beta, \mathrm{TNF} \alpha$, and IL-6, and reduce NF-KB-mediated proinflammatory signaling [21-24]. Furthermore, DMF and MMF can suppress vascular cell adhesion molecule 1 (VCAM-1) expression in endothelial cells in vitro $[25,26]$. In the experimental autoimmune encephalitis (EAE) mouse model, the administration of DMF reduces macrophage infiltration into areas of demyelination $[27,28]$, and in the LPS-induced neuroinflammation mouse model, DMF can reduce the expression of the microglial activation marker, Iba1 [29]. Based upon many such observations and human clinical therapeutic trials, oral DMF preparations have been approved for the treatment of multiple sclerosis [30,31]. The multiple immune-modulating effects of DMF have prompted investigations into its repurposing for use in other diseases that are associated with inflammation and oxidative stress, including HIV infection [32-35].

We previously identified the neuroprotective mechanistic effects of both DMF and MMF against HIV in our cell co-culture model containing both HIV-infected human macrophages and primary rodent neurons $[20,36]$. The HIV infection of macrophages results in a marked reduction in the expression of HO-1, which is associated with the release of neurotoxic levels of glutamate $[20,36]$. The exposure of these infected macrophages to DMF or MMF induces the expression of HO-1 and other ARE-driven enzymes and markedly reduces macrophage glutamate release, thus dramatically reducing excitotoxic neuronal injury. The selective induction of HO-1 expression in HIV-infected macrophages through targeted siRNA derepression confirms these HO-1 effects on glutamate release [36].

In this study, we determined whether DMF would induce antioxidant and antiinflammatory responses within the brains of rhesus macaques during SIV infection, which is the non-human primate model of HIV infection. Studies in various rodent models of neurodegeneration and neuroinflammation have shown that DMF administration results in antioxidative and anti-inflammatory effects in the rodent brain (reviewed in [37]), with excellent tissue penetration [38]. We used the rhesus macaque $\mathrm{CD} 8^{+} \mathrm{T}$ lymphocyte depletion model of pathogenic SIV infection, as this model shows consistent brain inflammation and oxidative stress associated with a relatively rapid progression (weeks) to severe SIVinduced immune deficiency [39]. We reasoned that this accelerated pathogenesis model would be better suited for assessing the CNS effects of DMF treatment than an SIV infection model without $\mathrm{CD} 8^{+} \mathrm{T}$ lymphocyte depletion, which is associated with less consistent brain inflammation and oxidative stress. Such consistent neuropathogenic responses are particu- 
larly important in studies that are limited to the relatively small numbers of macaques that can be resourced for therapeutic studies [40].

\section{Materials and Methods}

\subsection{Ethical Statement for Use of Nonhuman Primates}

This study was performed following the Institutional Animal Care and Use Committee (IACUC) of Tulane University approved protocol, number A3180-01, in accordance with the Animal Welfare Act and other federal statutes and regulations relating to animals. The animals were housed at the Tulane National Primate Research Center (TNPRC) at Tulane University (Covington, LA, USA) following the guidelines established by the National Institutes of Health (NIH) and under the supervision of the Association for the Assessment and Accreditation of Laboratory Animal Care (AAALAC)-accredited Division of Animal Resources.

\subsection{Administration of DMF to Rhesus Macaques}

Based upon the approved DMF daily dose of $480 \mathrm{mg}(\approx 7 \mathrm{mg} / \mathrm{kg})$ in humans, we synthesized gelatin-coated capsules (30 mg DMF [Sigma, St. Louis, MO, USA]/methyl cellulose matrix) for equivalent dosing in macaques. The capsules were prepared by the Investigational Drug Service at the University of Pennsylvania. Crystalline DMF was mixed with methylcellulose, pressed into $30 \mathrm{mg}$ capsules, and coated with gelatin (BSEfree, certified, pharmaceutical grade). To determine the DMF uptake in uninfected control macaques, DMF $(30 \mathrm{mg}$ ) embedded into FLAVORx medication flavoring (Patterson veterinary, Greeley, CO, USA) was administered by gastric tube or by oral ingestion (one macaque each). For comparison, one macaque received a DMF capsule administered via a gastric tube. We used a $30 \mathrm{mg}$ dose to analyze the single-dose kinetics of DMF absorption and metabolism to determine the lower expected peak plasma concentration of DMF dosing used in the study.

\subsection{Detection and Kinetics of MMF in Rhesus Macaque Plasma}

Blood was harvested at 0,60,120, 180, 240, 300, 360, and 480 min after the DMF administration. Plasma was assayed for MMF (Sigma, Inc.) via liquid chromatographymass spectrometry (LC-MS, Agilent 6490 Triple Quad LC-MS/MS; Agilent Technologies, Santa Clara, CA, USA) using a labeled MMF standard curve. The concentration of MMF was determined using LC-MS through the Childrens Hospital of Philadelphia Metabolics Core [41]. An eight-point standard curve (concentrations from 10,000 to $78 \mathrm{ng} / \mathrm{mL}$ ) was prepared by reconstituting MMF in macaque plasma, which was previously separated from untreated rhesus macaque blood, and detected using monomethyl fumarate- ${ }^{13} \mathrm{C}$ D3. Plasma from DMF-treated animals was separated and samples were extracted with acetonitrile, dried, and reconstituted in acetonitrile solution. Each sample was then assayed using LC-MS to establish the MMF content.

\subsection{DMF Treatment and SIV Infection}

Nine healthy, Indian rhesus macaques (from 5 to 17 years old) were used. Animals were divided into two groups: (i) SIV+ and (ii) SIV+/DMF treated (Figure 1). Enteral DMF was administered twice daily, $60 \mathrm{mg}$ in the morning and $30 \mathrm{mg}$ in the evening (daily treatment of $90 \mathrm{mg}, \approx 7 \mathrm{mg} / \mathrm{kg}$ of body weight), starting 7 days prior to the SIV $_{\text {mac251 }}$ intravenous (iv) inoculation (100 TCID 50 (50\% tissue culture infectious dose] in $1 \mathrm{~mL}$ of sterile phosphate-buffered saline). All macaques selected were male, as sex is a known biological variable in Nrf2-regulated genes [42] and anti-oxidant responses in males and females [43]. The availability of rhesus macaques at the Tulane National Primate Research Center allowed all male animals to be selected for this study to eliminate confounding sex effects on the targeted antioxidant responses. 
Treatment, infection, CD8 depletion and necropsy time points

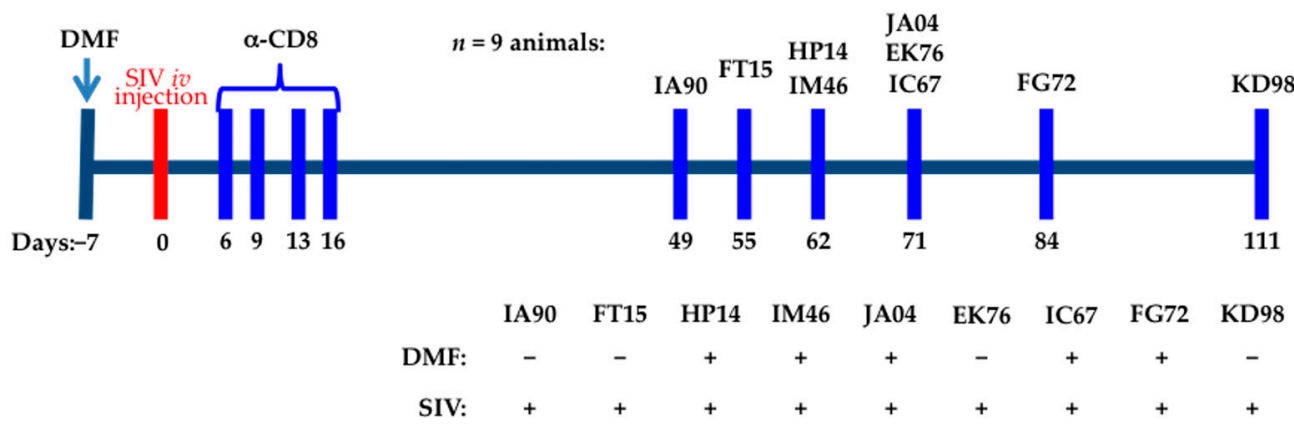

Figure 1. Schedule for dimethyl fumarate (DMF) treatment, anti-CD8 antibody treatments, simian immunodeficiency virus (SIV) inoculation, and necropsies of rhesus macaques. DMF treatment (90 mg total DMF daily, 5 animals DMF-treated, 4 untreated) was initiated seven days prior to the SIV infection, which was considered to be day " 0 ". On day " 0 " all animals (9 rhesus macaques) were intravenously infected with $100 \mathrm{TCID}_{50}\left(50 \%\right.$ tissue culture infectious dose) $\mathrm{SIV}_{\text {mac251. CD8 }}{ }^{+} \mathrm{T}$ lymphocyte depletion was achieved via treatment with anti-CD8 antibody infusions from 6 to 16 days post infection (dpi) via subcutaneous administration $(10 \mathrm{mg} / \mathrm{kg}$ for the first dose and then subsequent doses at $5 \mathrm{mg} / \mathrm{kg}$. Necropsies were performed at the indicated dpi thereafter. Individual animals are identified by unique letter-number combinations.

Animals were treated with the CD8+-T-lymphocyte-depleting antibody cM-T807 (provided by the Tulane Nonhuman Primate Reagent Resource Center) via subcutaneous injection, beginning at 6 days post infection (dpi) at a dose of $10 \mathrm{mg} / \mathrm{kg}$. Subsequent doses (5 mg/kg) were applied at 9, 13, and $16 \mathrm{dpi}$.

\subsection{Plasma and the Cerebrospinal Fluid (CSF) Viral Load}

Animals were infected with SIV $_{\text {mac251 via iv infusion (100 TCID }}$ ) [44]. The plasma and CSF samples were collected at time points up until the day of necropsy (Figure 1). The plasma and CSF were immediately frozen $\left(80^{\circ} \mathrm{C}\right)$. The plasma and CSF viral loads were determined using the Quantitative Molecular Diagnostics Core AIDS and Cancer Virus Program at the Frederick National Laboratory for Cancer Research (Leidos Biomedical Research, Frederick, MD, USA). The presence of SIV RNA was determined with a sensitive (15 copies Eq/mL threshold sensitivity), quantitative real-time polymerase chain reaction (qPCR) assay that targets a region in SIV gag using primers and a TaqMan ${ }^{\circledR}$ probe (ThermoFisher Scientific, Waltham, MA, USA). The following primer pairs were used: forward primer (SGAG21) 5'-GTCTGCGTCATPTGGTGCATTC-3' and reverse primer (SGAG22) 5'-CACTAGKTGTCTCTGCACTATPTGTTTTG-3', along with probe (pSGAG23) 5'-(FAM) CTTCPTCAGTKTGTTTCACTTTCTCTTCTGCG-(BHQ $\left.{ }^{\mathrm{TM}} 1\right)-3$ [45].

\subsection{Brain and Peripheral Tissue Harvesting}

Eleven brain regions were harvested at the necropsy time points (frontal cortex, third ventricle, thalamus, parietal cortex, subcortical white matter, cerebellum, brainstem, basal ganglia, caudate nuclei, temporal cortex, and occipital cortex). Thymus, liver, and spleen tissues were also collected. All tissues for Western blot or RNA analysis were immediately frozen $\left(80^{\circ} \mathrm{C}\right)$. Tissues for immunohistochemistry (IHC) staining and optical redox imaging were preserved in paraformaldehyde for paraffin embedding.

\subsection{Western Blotting}

Tissue lysates were prepared using homogenization $(\approx 100 \mathrm{mg}$ of tissue) via silica bead beating and sonication in a seven-volumes buffer (weight/volume) (10 mM Tris- $\mathrm{HCl} \mathrm{pH} 7.8$, $0.5 \mathrm{mM}$ dithiothreitol, $5 \mathrm{mM} \mathrm{MgCl} 2,0.03 \%$ Triton X-100) containing a phosphatase inhibitor cocktail (PhosSTOP, Roche Diagnostics GmbH, Mannheim, Germany) and a protease inhibitor cocktail (cOmplete, Roche Diagnostics $\mathrm{GmbH}$ ). The protein was quantified using the DC ${ }^{\text {TM }}$ (detergent compatible) protein assay (Bio-Rad Laboratories, Hercules, CA, USA). Equivalent amounts of proteins were added to a Laemmli sample buffer (Sigma, Inc.) 
with 2.5\% 2-mercaptoethanol (Bio-Rad laboratories) and then denatured at $95{ }^{\circ} \mathrm{C}$ for $10 \mathrm{~min}$. Proteins were resolved on an SDS-PAGE gel and transferred overnight at $4{ }^{\circ} \mathrm{C}$ to poly(vinylidene fluoride) (PVDF) membranes (Merck Millipore Ltd., Carrigtwohill, Co. Cork, Ireland). The membranes were blocked with an Odyssey Blocking Buffer (PBS) (LI-COR Biosciences, Lincoln, NE, USA) and incubated with primary antibody overnight $\left(4{ }^{\circ} \mathrm{C}\right)$ (Table 1 for the list of antibodies). TRDye-conjugated secondary antibodies (LICOR Biosciences) were used to detect the primary antibody. Background-corrected signal quantification of protein bands was determined using Image Studio Lite software (LI-COR Biosciences). One sample prepared from mixing equal volumes of all samples (Mix) was used as a running and transfer control in all membranes. Each blot was normalized to that sample in each membrane, allowing for comparisons between all brain regions and animals.

Table 1. Information regarding the antibodies used in the Western blotting and immunohistochemistry (IHC).

\begin{tabular}{|c|c|c|c|c|c|c|}
\hline Antibody & Host & Isotype & Molecular Size & Dilution & Cat. No & Company \\
\hline Anti-HO-1 & Rabbit & Polyclonal & $31 \mathrm{kDa}$ & $1: 500$ & SPA-894 & $\begin{array}{c}\text { ENZO Life } \\
\text { Sciences }\end{array}$ \\
\hline Anti-HO-2 & Mouse & Monoclonal IgG2a & $36 \mathrm{kDa}$ & $1: 1000$ & MA5-25749 & ThermoFisher \\
\hline Anti-PRDX1 & Rabbit & Monoclonal & $22 \mathrm{kDa}$ & $1: 1000$ & 8732 & CST \\
\hline Anti-NQO1 & Mouse & Monoclonal IgG1 & $32 \mathrm{kDa}$ & $1: 5000$ & ab28947 & Abcam \\
\hline Anti-GPX1 & Rabbit & Monoclonal & $22 \mathrm{kDa}$ & $1: 2000$ & $3286 \mathrm{~S}$ & CST \\
\hline Anti-GFAP & Mouse & Monoclonal IgG1 & $48 \mathrm{kDa}$ & 1:2000 & $3670 S$ & CST \\
\hline Anti-VCAM-1 & Rabbit & Monoclonal & $81 \mathrm{kDa}$ & $1: 1000$ & ab134047 & Abcam \\
\hline Anti-ICAM-1 & Rabbit & Monoclonal & $58 \mathrm{kDa}$ & 1:1000 & ab109361 & Abcam \\
\hline Anti-PSD-95 & Mouse & Monoclonal IgG2a & $95 \mathrm{kDa}$ & 1:1000 & MAB1596 & Sigma, Inc. \\
\hline $\begin{array}{c}\text { Anti- } \\
\text { synaptophysin }\end{array}$ & Mouse & Monoclonal IgG1 & $37 \mathrm{kDa}$ & $1: 1000$ & ab8049 & Abcam \\
\hline Anti-synapsin 1 & Rabbit & Monoclonal & $75 \mathrm{kDa}$ & 1:1000 & $5297 \mathrm{~S}$ & CST \\
\hline Anti- $\beta$-tubulin & Mouse & Monoclonal IgG2b & $50 \mathrm{kDa}$ & 1:10000 & $86298 \mathrm{~S}$ & CST \\
\hline Anti-NFL & Rabbit & Monoclonal & $66 \mathrm{kDa}$ & 1:10000 & ab52989 & Abcam \\
\hline Anti- $\beta$-tubulin & Rabbit & Monoclonal & $50 \mathrm{kDa}$ & 1:3000 & $2128 \mathrm{~S}$ & CST \\
\hline Anti- $\beta$-actin & Mouse & Monoclonal IgG1 & $42 \mathrm{kDa}$ & $1: 30000$ & A4441 & Sigma, Inc. \\
\hline Anti-8-OHdG ${ }^{1}$ & Mouse & Monoclonal IgG1 & $\mathrm{N} / \mathrm{A}$ & 1:100000 & $a b 48508$ & Abcam \\
\hline Anti-3NT ${ }^{1}$ & Mouse & Monoclonal IgG2a & $\mathrm{N} / \mathrm{A}$ & $1: 2500$ & ab61392 & Abcam \\
\hline Anti-HLA-DR ${ }^{1}$ & Mouse & Monoclonal IgG2b & $\mathrm{N} / \mathrm{A}$ & $1: 500$ & $14-9956-82$ & ThermoFisher \\
\hline Anti-CD68 ${ }^{1}$ & Mouse & Monoclonal IgG3 kappa & $\mathrm{N} / \mathrm{A}$ & $1: 200$ & M087629-2 & $\begin{array}{c}\text { Agilent } \\
\text { Technologies }\end{array}$ \\
\hline
\end{tabular}

ENZO Life Sciences (Farmingdale, NY, USA). Abcam (Cambridge, United Kingdom). CST: Cell Signaling Technologies (Danvers, MA, USA). N/A: not applicable; ${ }^{1}$ used for IHC.

\subsection{Immunohistochemistry Analyses}

One of two adjacent portions of each brain region per animal was fixed via immersion in $4 \%$ paraformaldehyde for $48 \mathrm{~h}$, washed in PBS, and transferred to $70 \%$ ethanol before paraffin embedding and sectioning $(8 \mu \mathrm{m})$. The remaining portion was frozen and processed for protein quantification, as described above. For the IHC staining, four sections from each region were harvested from each animal for labeling with each antibody. Sections $(8 \mu \mathrm{m})$ were deparaffinized in xylene and rehydrated in ethanol and distilled water, quenched with $3 \% \mathrm{H}_{2} \mathrm{O}_{2}$ for $30 \mathrm{~min}$, and microwaved for $15 \mathrm{~min}$ in a $10 \mathrm{mM}$ citrate buffer ( $\mathrm{pH}$ 6) (Vector Laboratories, Inc., Burlingame, CA, USA) for epitope exposure. Primary antibodies (Table 1) and isotype-matched control antibodies were used at identical concentrations for staining with an overnight incubation $\left(4^{\circ} \mathrm{C}\right)$, two successive washes, and incubation with an appropriate biotin-conjugate secondary antibody. The signal was amplified using an avidin-biotin horseradish peroxidase system following the manufacturer's instructions (Vectastain ABC kit, Vector Laboratories, Inc.). A chromophore reaction was developed with diaminobenzidine and hydrogen peroxide. All slides from both groups of animals (untreated vs. treated) and for each marker were stained at the same time and with the same batch of reagents. The sections were dehydrated and mounted 
with Cytoseal 60 (ThermoFisher). Images from all sections were taken using a digital slide scanner (Aperio AT2, Leica Biosystem, Wetzlar, Germany) at 20x magnification and were analyzed with QuPath software (version 0.2.0-m5, University of Edinburgh, Edinburgh, United Kingdom), as previously reported [46].

\subsection{Optical Redox Imaging}

The autofluorescence of protein-bound NADH and oxidized flavoproteins containing flavin adenine dinucleotide (Fp) of formalin-fixed-paraffin-embedded (FFPE) tissue slides $(8 \mu \mathrm{m})$ was imaged and quantified, as previously described [47]. NADH signals were collected using bandpass filters with excitation (Ex) at 370-400 nm, and emission (Em) at 414-450 nm. The Fp signals were acquired with Ex: 450-488 nm and Em: 500-530 nm bandpass filters using a Zeiss wide-field microscope (Axio Observer.Z1/7, One North Broadway, White Plains, NY, 10601, USA). Tile images (pixel size $1.172 \times 1.172 \mu \mathrm{m}^{2}$ ) were taken using a $5 \times / 0.16$ NA objective with shading correction on the fly, followed by photostitching. The acquired images were processed with a customized Matlab ${ }^{\circledR}$ (The MathWorks, Inc., 1 Apple Hill Dr, Natick, MA 01760, USA) routine to generate the redox images where the redox ratio $\mathrm{Fp} /(\mathrm{NADH}+\mathrm{Fp})$ image was generated pixel-by-pixel from the NADH and Fp images and to obtain the mean value of each of the redox indices (NADH, Fp, the redox ratio) using global averaging [47-49]. Obvious fluorescent artifacts (in either channel) were removed during the imaging analysis.

\subsection{Plasma and CSF Neurofilament Assay}

The plasma and CSF collected from each animal at its necropsy time point were used to quantify NFL using a Singleplex Assay (Meso Scale Discovery, Rockville, MD, USA), as described in the R-PLEX ${ }^{\mathrm{TM}}$ protocol and as previously reported [6].

\subsection{Data Acquisition and Statistical Analyses}

Statistical analyses were performed using GraphPad Prism software (San Diego, CA, USA). Data were analyzed using unpaired and paired $t$-tests.

\section{Results}

3.1. Enteral Administration of a Human-Equivalent Therapeutic DMF Dose in Rhesus Macaques Produced Therapeutic Plasma Levels of MMF

Previous studies have confirmed the detection of MMF, and not DMF, in the plasma, serum, and whole blood of DMF-treated individuals [50-52]. Such a conversion of DMF to MMF occurs very rapidly (minutes) through hydrolysis-mediated intestinal and serum hydrolases [52]. To confirm the absorption of DMF in rhesus macaques, we administered a single DMF dose (30 mg) in each of three ways: (i) gastric tube delivery of a gelatincoated capsule (DMF admixed with methylcellulose), (ii) oral ingestion of DMF mixed in a foodstuff, and (iii) gastric tube delivery of a DMF suspension (Figure 2). DMF treatments were continued up to the day of elective euthanasia and necropsy. Using LC-MS analysis, we detected plasma MMF at concentrations up to $4.1 \mu \mathrm{M}$ at $60 \mathrm{~min}$ with each method of administration, followed by reduced plasma levels at 120 and $240 \mathrm{~min}$. As previously reported, only MMF, and not DMF (not shown), was detected. This plasma MMF level was within the therapeutic plasma level range in humans treated for multiple sclerosis with oral DMF preparations [50,51]. We, therefore, implemented a treatment regimen of twice-daily DMF administration (similar to human regimens) of gelatin-encapsulated DMF (30 mg, $60 \mathrm{mg}$ ). 
(A)

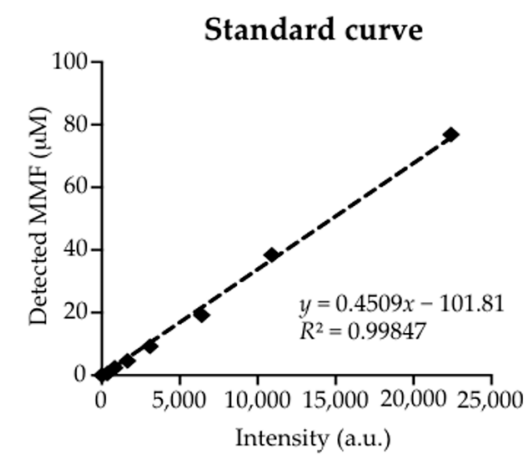

(B)

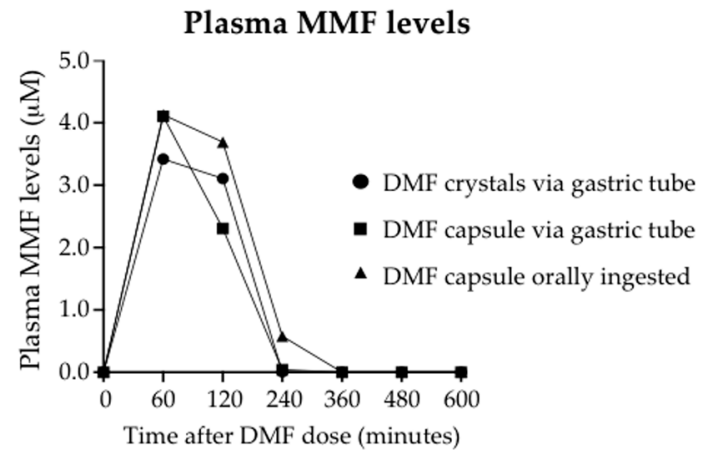

Figure 2. Plasma monomethyl fumarate (MMF) levels in rhesus macaques after a single enteral dose of DMF (30 mg). (A) An eight-point standard curve was prepared using serial dilutions (MMF concentrations from 77 to $0.6 \mu \mathrm{M}$ ). MMF was reconstituted in macaque plasma, which was previously separated from untreated rhesus macaque blood, and detected using liquid chromatography-mass spectrometry. (B) Each of three adult male rhesus macaques $(8.1 \mathrm{~kg}, 9.1 \mathrm{~kg}, 11.4 \mathrm{~kg})$ received a single $30 \mathrm{mg}$ dose of DMF admixed in methylcellulose, either as a gelatin-coated capsule or as a crystalline suspension. One animal received the DMF capsule via a gastric tube. One animal received $30 \mathrm{mg}$ of DMF embedded in a foodstuff, which was orally ingested. The remaining animal received a suspension of DMF crystals via a gastric tube. Plasma was separated from whole blood drawn at each indicated time point after the DMF administration. Samples were extracted with acetonitrile, dried, and reconstituted in acetonitrile solution. Each sample was assayed using LC-MS to establish the MMF content. Standard curves were prepared using monomethyl fumarate- ${ }^{13} \mathrm{C}-\mathrm{D} 3$ for calibration.

\subsection{Plasma and Cerebrospinal Fluid SIV Levels Were Not Altered by the DMF Treatment}

The DMF treatment was initiated one week prior to the SIV infection, followed by $\mathrm{CD}^{+} \mathrm{T}$ lymphocyte depletion (Figure 1) and monitoring of the plasma and CSF viral loads (Figure 3). Plasma SIV was detected at 6 dpi in all animals (Figure 3A), while CSF SIV was detected in one animal at $6 \mathrm{dpi}$ and all but one animal at $21 \mathrm{dpi}$ (Figure 3B). At the necropsy time points, CSF SIV was detected in all animals. Over the course of the infection, the plasma SIV levels were approximately $1 \log$ higher than those in CSF, as has previously been reported [53]. There was no significant difference between the SIV levels in DMF-treated vs. non-treated animals, which is consistent with our prior data showing no effect of DMF or MMF on HIV replication in HIV-infected macrophages [4].

\subsection{DMF Treatment Induced Nrf2/ARE-Driven Gene Expression Throughout the Brain}

DMF targets the Nrf2/ARE pathway, which involves multiple genes that protect cells from oxidative stress and injury [37,54-59], including antioxidant enzymes, such as NQO1 [60], GPX1 [61], PRDX1 [62], and HO-1 [63]. We quantified the antioxidant enzyme expression in eleven brain regions (Figure ), as well as the spleen (Figure 5), thymus, and liver (Figure S1), and observed higher NQO1 $(p<0.01)$, GPX1 $(p<0.001)$, and HO-1 $(p<0.05)$ expression in the brains of DMF-treated animals compared to untreated animals (via comparing the means of expression from each region in each group using a paired $t$-test, Figure $\mathrm{B}, \mathrm{D}, \mathrm{F}, \mathrm{H}, \mathrm{J})$. Individual regional changes associated with DMF treatment were observed only for GPX1 expression in the frontal cortex, third ventricle, thalamus, parietal cortex, basal ganglia, and caudate nucleus (Figure C,D). HO-2 expression was unchanged with DMF treatment in all brain regions analyzed (Figure I,J). Unlike the DMF treatment effects in the brain, the DMF treatment effects in the spleen were associated with higher expressions of PRDX1 and HO-2 and no changes in NQO1, GPX1, or HO-1 (Figure 5A-F, unpaired $t$-test, $p<0.01)$. No changes in any of these enzymes were observed in the liver or thymus (Figure S1). 
(A) Plasma viral loads

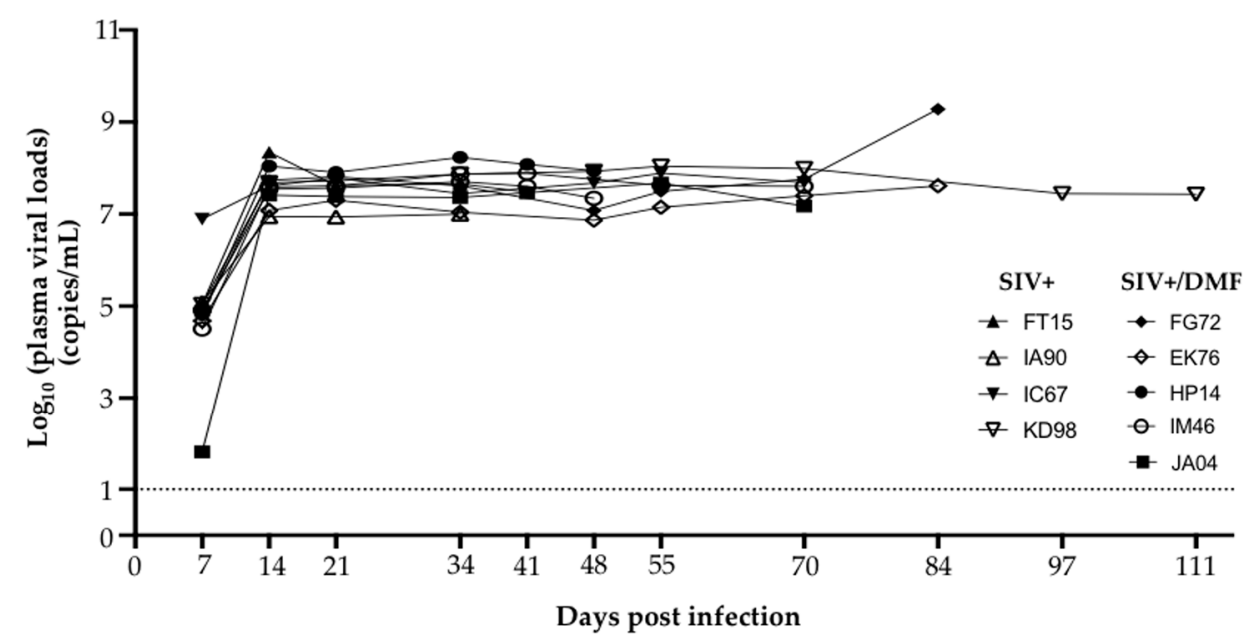

(B) CSF viral loads

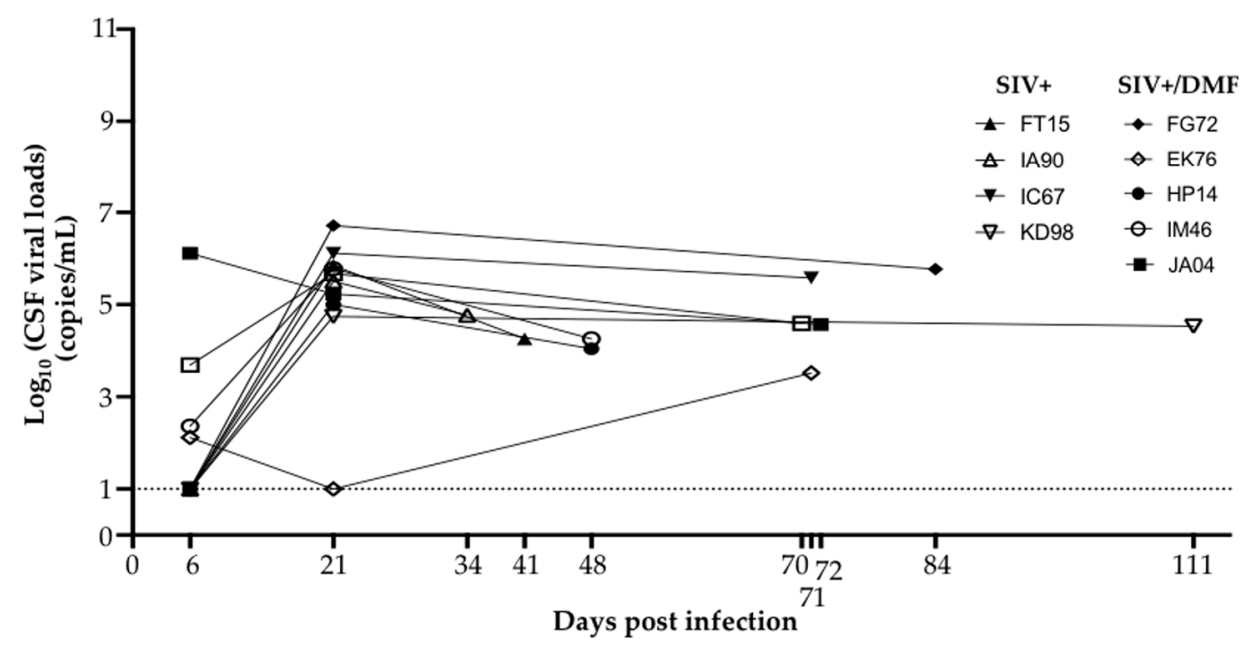

Figure 3. SIV RNA was detectable in plasma from all animals after $7 \mathrm{dpi}$ and in cerebrospinal fluid (CSF) at the necropsy time points. (A) SIV plasma viral loads (RNA copies $/ \mathrm{mL}$ ) from each animal. SIV RNA was detectable from 7 dpi until the necropsy day. (B) The SIV CSF viral loads (RNA copies $/ \mathrm{mL}$ ) from each animal. SIV RNA was detectable in one animal at 6 dpi. One animal did not have detectable CSF SIV RNA until the necropsy day. Nine rhesus macaques, all SIV-infected, were used in the study (4 untreated animals and 5 DMF-treated ( $90 \mathrm{mg}$ total DMF daily)). Each dot represents the value for each animal at each time point. Individual animals are listed by unique letter-number combinations. 

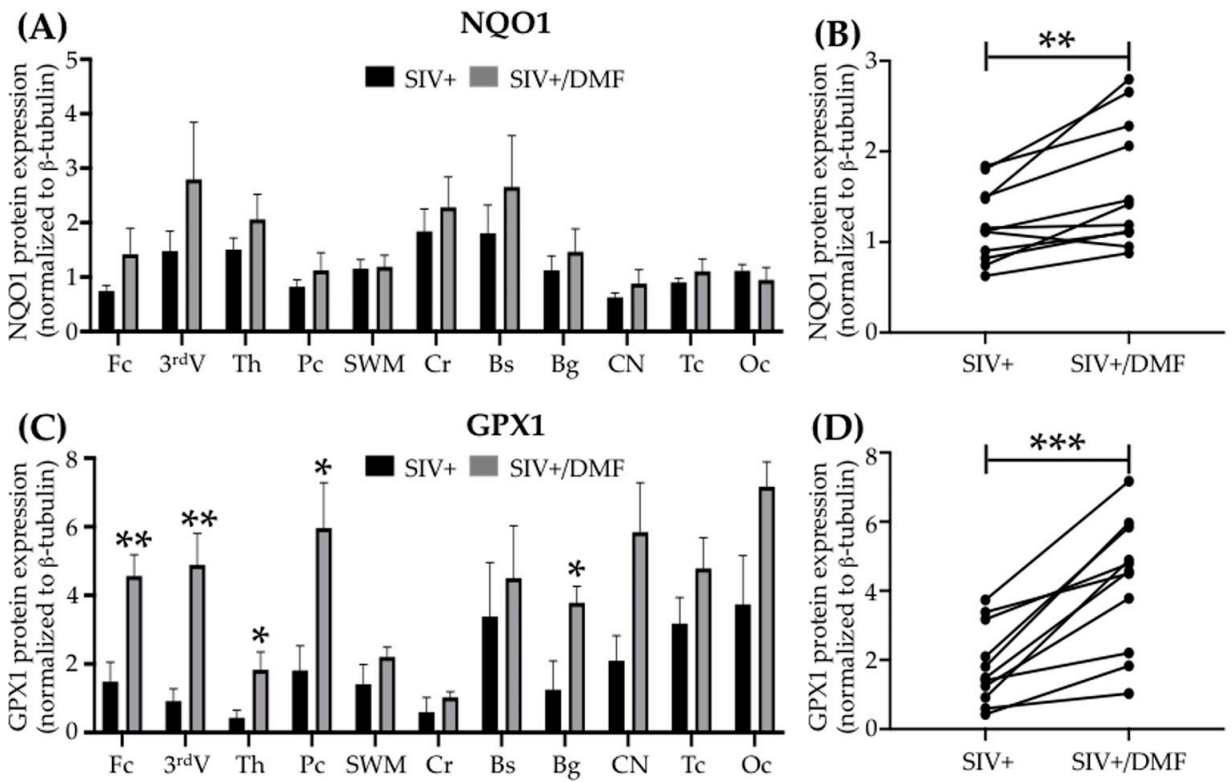

(E)

PRDX1

(F)
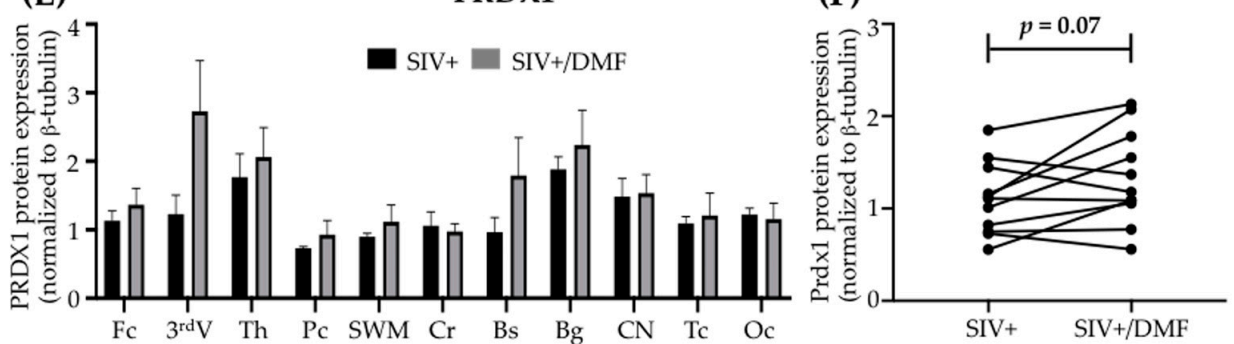

(G)

HO-1

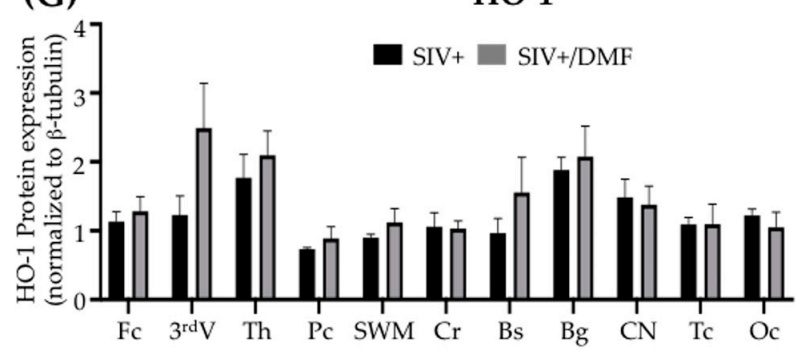

(H)

(I)

HO-2
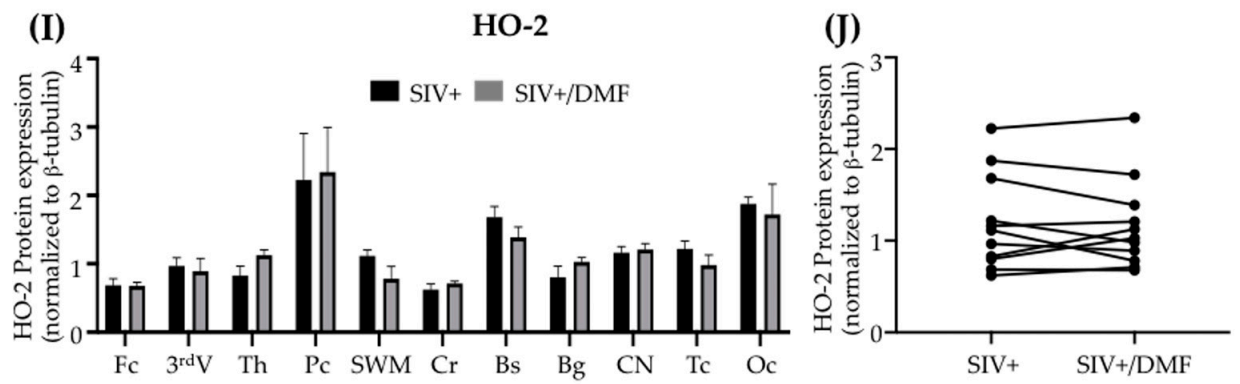

Figure 4. DMF treatment was associated with higher antioxidant enzyme expression in the brains of SIV-infected macaques. (A) No significant difference in NQO1 expression was observed in the individual regions using Student's unpaired $t$-test (Fc: frontal cortex, $3^{\text {rd }} \mathrm{V}$ : third ventriculi, $\mathrm{Th}$ : thalamus, Pc: parietal cortex, SWM: subcortical white matter, Cr: cerebellum, Bs: brainstem, Bg: basal ganglia, $\mathrm{CN}$ : caudate nuclei, Tc: temporal cortex, Oc: occipital cortex). (B) A significant increase in overall mean NQO1 expression was observed with DMF treatment (paired $t$-test). (C) A significant increase in GPX1 expression was observed in the $\mathrm{Fc}, 3^{\text {rd }} \mathrm{V}$, Th, Pc, and Bg with DMF treatment. (D) A 
significant increase in overall mean GPX1 expression was observed with DMF treatment. (E) No significant difference in PRDX1 expression was observed in individual regions. (F) A statistically non-significant $(p=0.07$ ) increase in the overall mean PRDX1 expression was observed with DMF treatment. (G) No significant difference in HO-1 expression was observed in individual regions. (H) A significant increase in overall mean HO-1 expression was observed with DMF treatment. (I,J) No significant difference in HO-2 expression was observed in individual regions or overall in the brains with DMF treatment. Differences in expression within individual brain regions between both groups (DMF-treated and untreated) were evaluated using Student's unpaired $t$-test and comparison between the mean of the expression for each enzyme in each group was done using Student's paired $t$-test $\left({ }^{*} p<0.05,{ }^{* *} p<0.01,{ }^{* * *} p<0.001\right)$. Nine SIV-infected rhesus macaques (4 untreated animals and 5 DMF-treated (90 mg total DMF daily)). All quantifications are expressed as mean \pm standard error of the mean (SEM). In the right panels, each dot represents the average for each region and group.

(A)

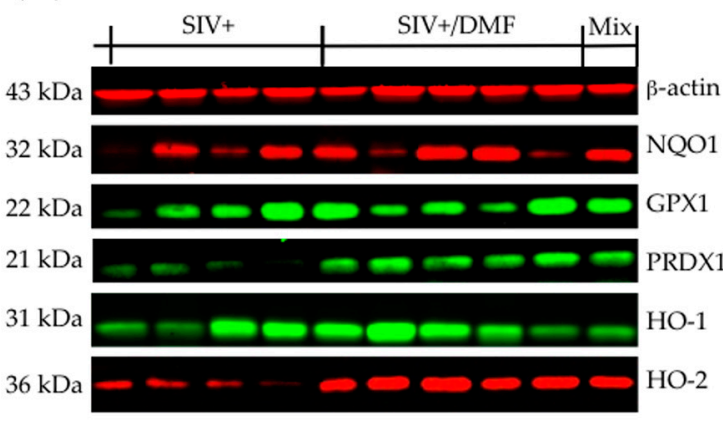

(C)

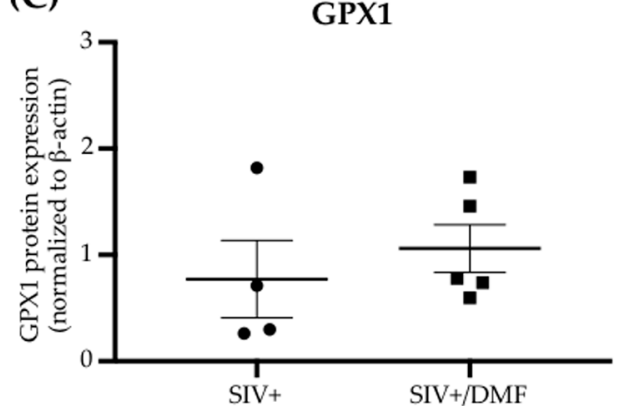

(E)

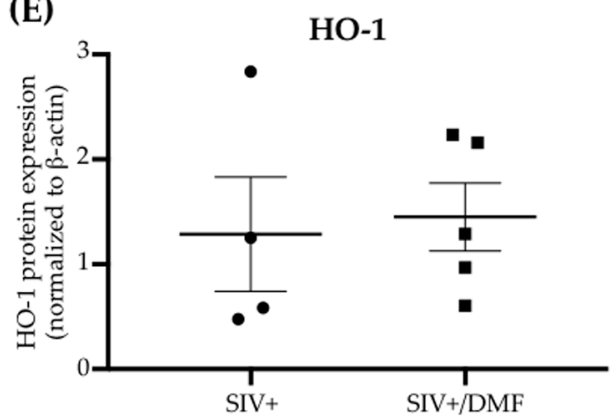

(B)

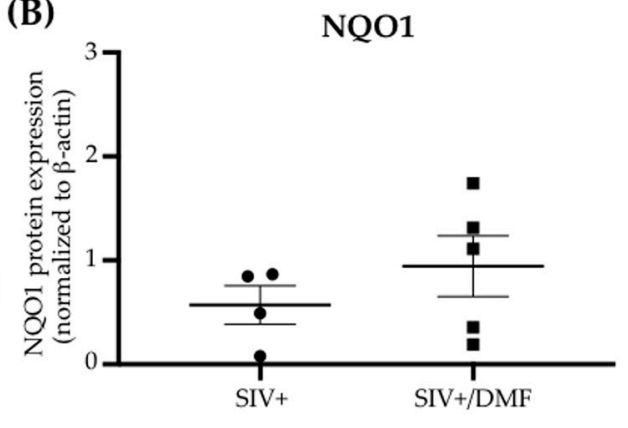

(D)

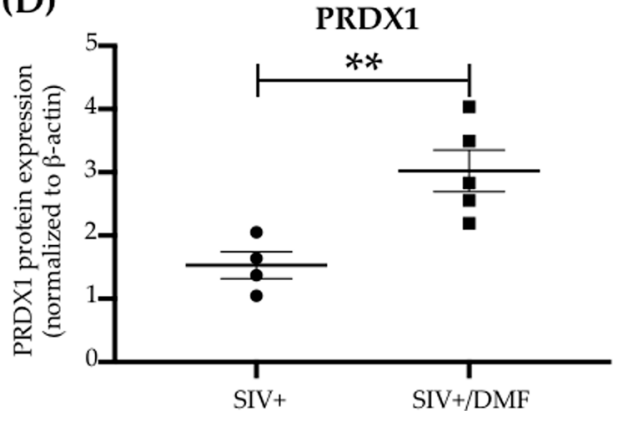

(F)

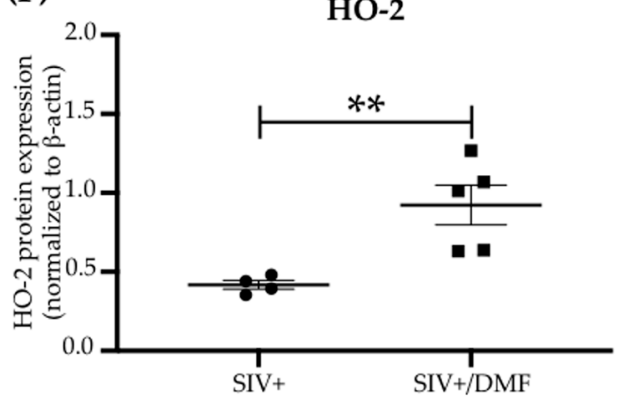

Figure 5. DMF treatment was associated with higher antioxidant enzyme expression in the spleens of SIV-infected macaques. (A) Representative immunoblot for the antioxidant enzymes assessed. $\beta$-Actin was used as a loading control. (B,C) No significant differences in the expression of NQO1 and GPX1, respectively, were observed in the spleens with DMF treatment. (D) A significant increase in the PRDX1 expression was observed with DMF treatment. (E) No significant difference in the expression of HO-1 was observed with DMF treatment. (F) A significant increase in the HO-2 expression was observed with DMF treatment. Spleen samples from nine SIV-infected rhesus macaques (4 untreated animals and 5 DMF-treated (90 mg total DMF daily)) were analyzed using Western blotting. Each dot represents the quantified value per animal. Statistical analyses were done using Student's unpaired $t$-test, ${ }^{* *} p<0.01$. All values are expressed as mean $\pm \mathrm{SEM}$. 


\subsection{DMF Treatment Was Associated with a Lower Expression of DNA and Protein Oxidation Markers and a More Reduced Brain Redox State}

We applied a complementary approach of immunohistochemical labeling and optical redox imaging in sections from the frontal cortex and brainstem to determine the levels of oxidized proteins (3NT) and DNA (8-OHdG) (Figure 6) and the redox state (Figure 7; Figure 8). Notably, higher levels of protein (Figure 6A,B) and DNA (Figure 6C,D) oxidation were observed in the brainstem compared to the frontal cortex. Furthermore, DMF treatment was associated with lower protein oxidation in the brainstem (Figure 6A, $p<0.05$ ) and lower DNA oxidation in both regions (frontal cortex $p<0.001$ and brainstem $p<0.01$ ) (Figure 6C,D).

(A)

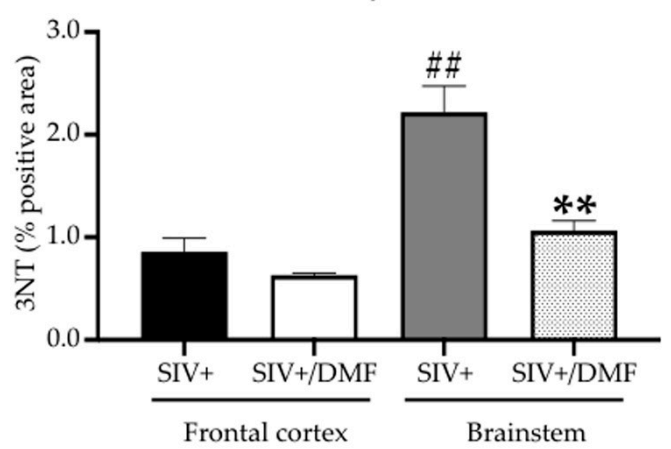

(B)

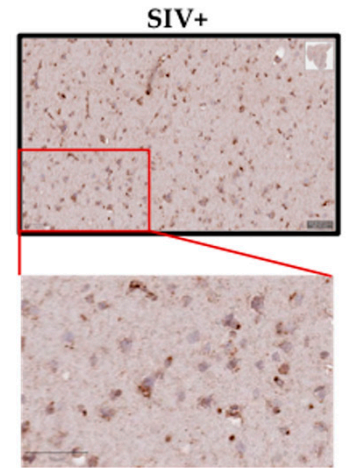

Frontal cortex

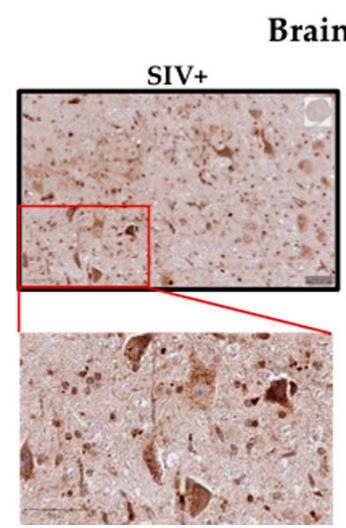

Brainstem
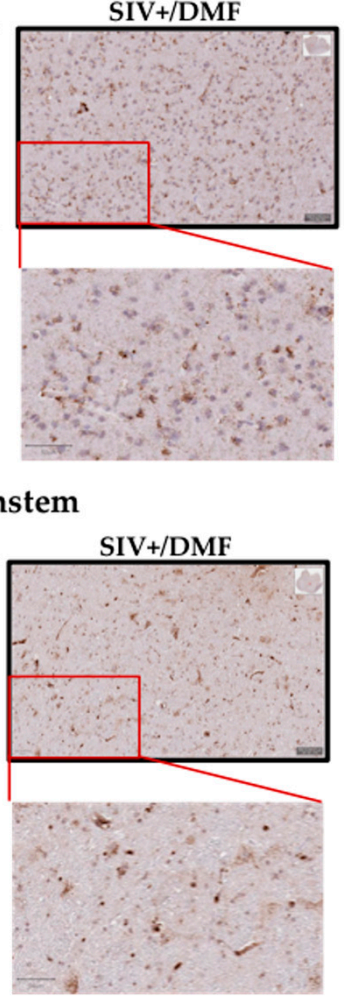

(C)

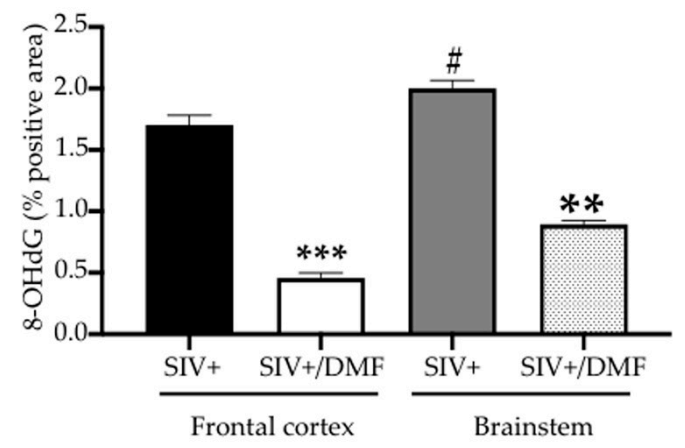

(D)

\section{Frontal cortex}
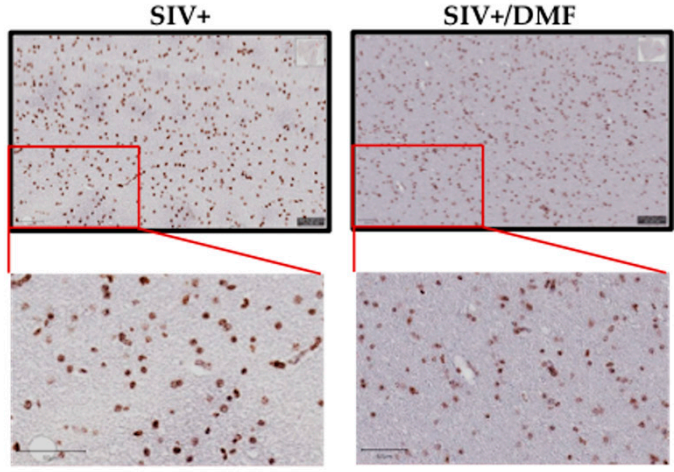

Brainstem
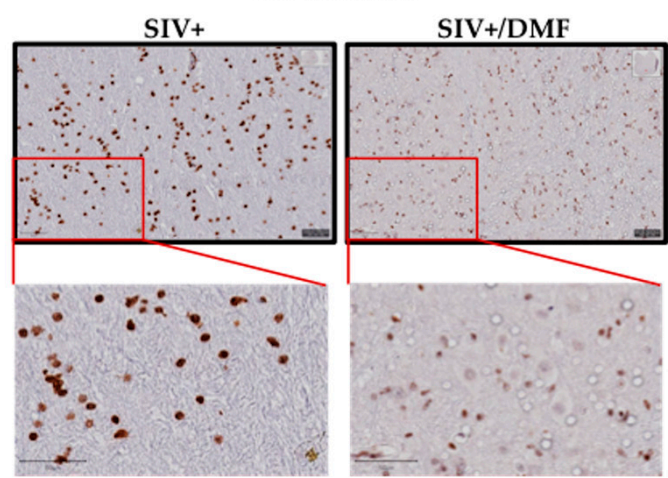

Figure 6. DMF treatment was associated with the reduced expression of markers of DNA and protein oxidation in the frontal cortexes and brainstems of SIV-infected macaques. (A) Quantification of 3NT (oxidized proteins) in the frontal cortex (left) and brainstem (right) showed reduced expression in the brainstem with DMF treatment. The 3NT labeling was significantly higher in the brainstem compared to the frontal cortex in SIV infection (statistical analysis was done with Student's 
unpaired $t$-test, ${ }^{* *} p<0.01$ comparing SIV+ vs. SVI+/DMF in the brainstem; ${ }^{\# \#} p<0.01$ comparing the frontal cortex vs. brainstem in the SIV+ group). (B) Representative labeling for 3NT in the frontal cortex (top panels) and brainstem (bottom panels). (C) Quantification of 8-OHdG (oxidized DNA) in the frontal cortex (left) and brainstem (right) showed reduced expression in the frontal cortex and brainstem with DMF treatment. 8-OHdG labeling was significantly higher in the brainstem compared to the frontal cortex in SIV infection (statistical analysis was done with Student's unpaired $t$-test, ${ }^{* *} p<0.01$ and ${ }^{* * *} p<0.001$ comparing SIV+ vs. SVI+/DMF; ${ }^{*} p<0.05$ comparing the frontal cortex to the brainstem in the SIV+ group). (D) Representative labeling for 8-OHdG in the frontal cortex (top panels) and brainstem (bottom panels). Four sections from each region and animal were used to be labeled with each antibody. Nine SIV-infected rhesus macaques were used in the study (4 untreated animals and 5 DMF-treated (90 mg total DMF daily)). Images from all sections were taken using a digital slide scanner (Aperio AT2, Leica Biosystem, Wetzlar, Germany) at 20x magnification and were analyzed with QuPath software (version 0.2.0-m5), as previously reported [46]. Quantifications are expressed as the mean percentage of positive staining from all the stained areas \pm SEM.
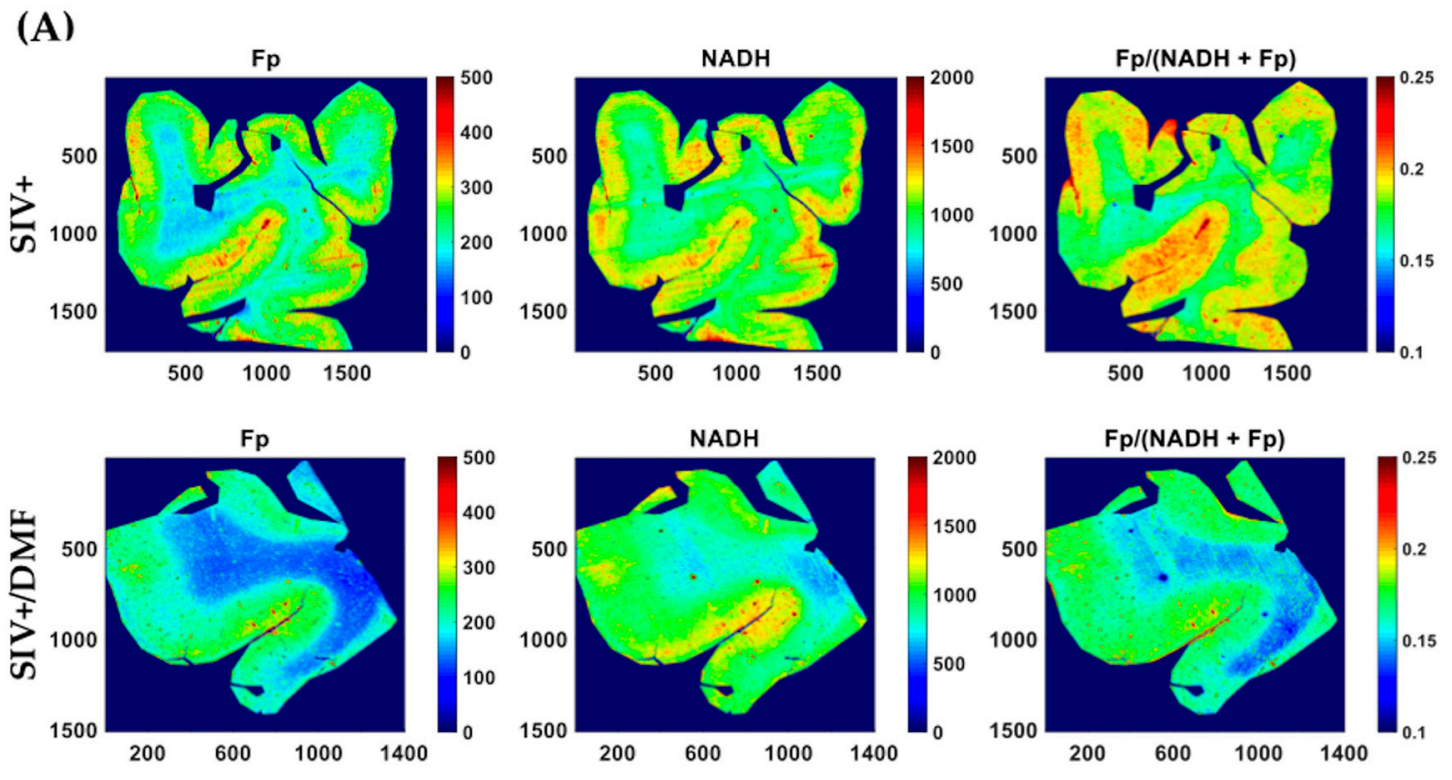

(B)

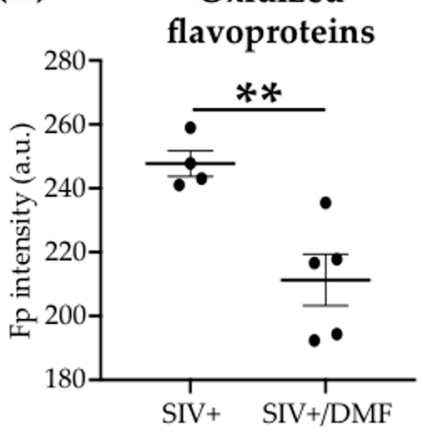

(C)

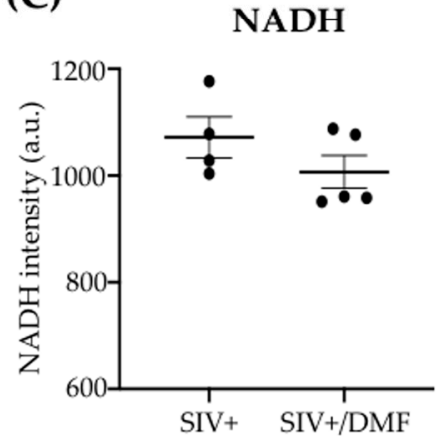

(D)

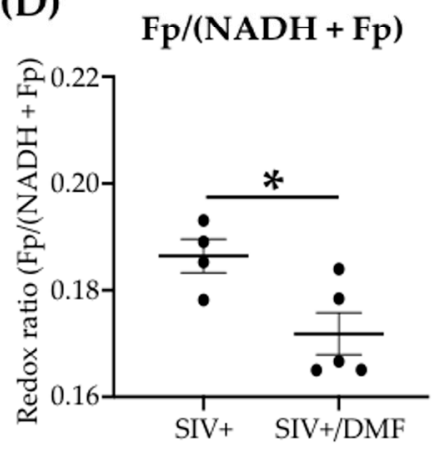

Figure 7. DMF treatment was associated with lower oxidized flavoprotein (Fp) levels and redox ratio Fp/(NADH + Fp) in the frontal cortex of SIV-infected macaques. (A) Representative images (frontal cortex) of autofluorescence of Fp (left), $\mathrm{NADH}$ (center) and Fp/(NADH + Fp) (right). The " $x$ " and " $y$ " coordinates of the image matrices are shown. Numbers and color bars on the right of each image indicate the range of the index being displayed. (B) Quantification of the Fp autofluorescence showed lower Fp levels with DMF treatment (statistical analysis was done with Student's unpaired t-test, $\left.{ }^{* *} p<0.01\right)$. (C) No significant difference in NADH levels was observed with DMF treatment. (D) Quantification of $\mathrm{Fp} /(\mathrm{NADH}+\mathrm{Fp})$ showed a more reduced redox ratio being associated with DMF treatment, which was indicative of the oxidative stress level (statistical analysis was done using Student's unpaired $t$-test, ${ }^{*} p<0.05$ ). Nine SIV-infected rhesus macaques were used in the study (4 untreated animals and 5 DMF-treated ( $90 \mathrm{mg}$ total DMF daily)). Intensities are in arbitrary units (a.u.) and all redox indices are expressed as the mean $\pm \mathrm{SEM}$. 

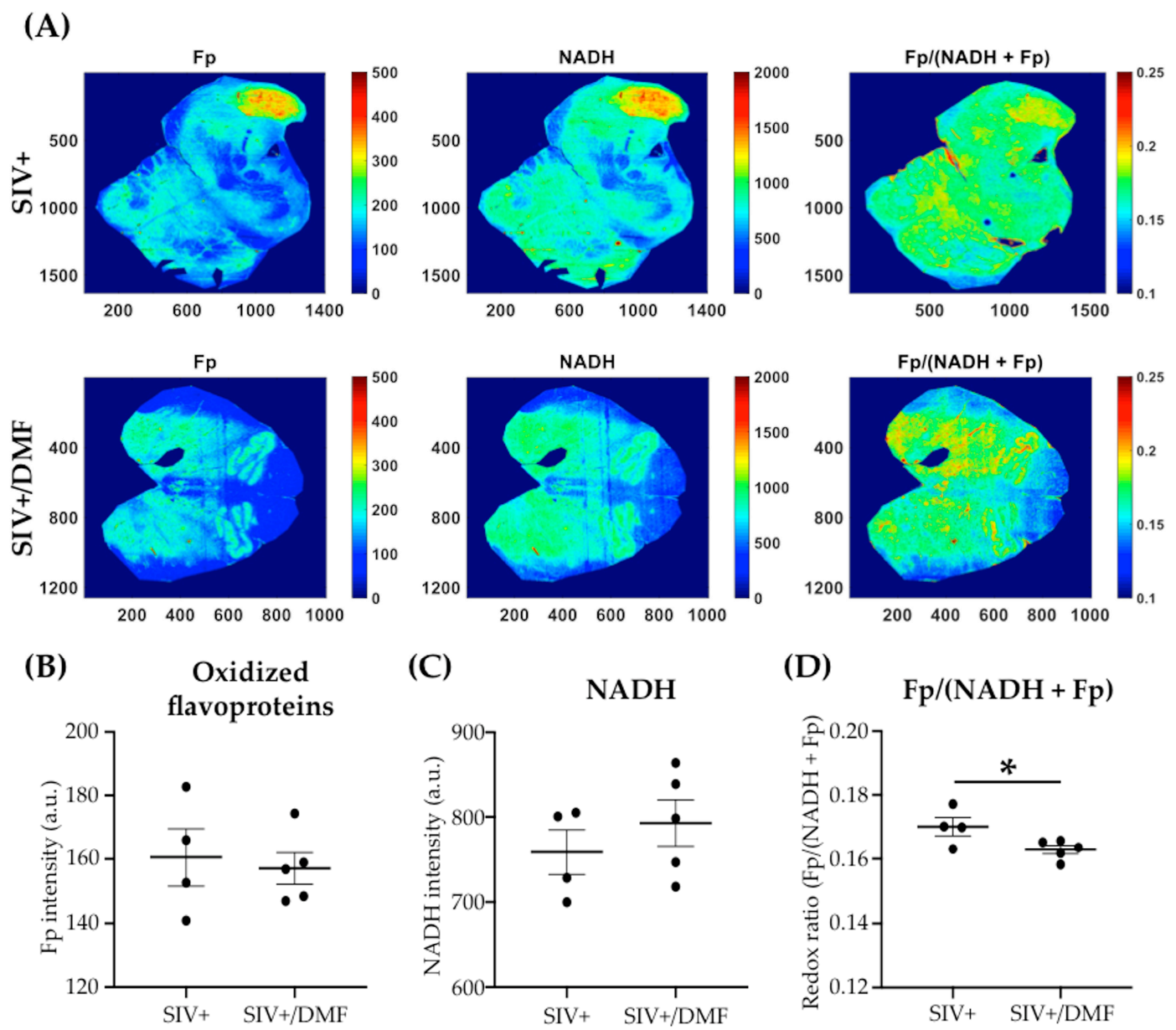

Figure 8. DMF treatment was associated with a lower redox ratio $\mathrm{Fp} /(\mathrm{NADH}+\mathrm{Fp})$ in the brainstem of SIV-infected macaques. (A) Representative images (brainstem) of the autofluorescence of Fp (left), NADH (center), and Fp/(NADH + Fp) (right). The " $x$ " and " $y$ " coordinates of the image matrices are shown. Numbers and color bars on the right of each image indicate the range of the redox index being displayed. (B) Quantification of the Fp autofluorescence showed no significant change in Fp levels with DMF treatment (statistical analysis was done with Student's unpaired $t$-test). (C) No significant difference in NADH levels was observed with DMF treatment. (D) Quantification of Fp/(NADH + Fp) showed that the DMF treatment was associated with a lower redox ratio, which was indicative of a lower oxidative stress level (statistical analysis was done with Student's unpaired $t$-test, $\left.{ }^{*} p<0.05\right)$. Nine SIV-infected rhesus macaques were used in the study (four untreated animals and five DMF-treated (90 mg total DMF daily)). Intensities are in arbitrary units (a.u.) and all redox indices are expressed as the mean \pm SEM.

To determine the effects of DMF treatment on the brain redox status, we quantified the autofluorescence of protein-bound NADH and Fp using the optical redox imaging of FFPE tissue slide technique [47]. NADH and Fp signals reflect the mitochondrial redox state and can detect the tissue redox contrast between different physiological/pathological states and therapeutic responses $[64,65]$. Within the frontal cortex, we observed lower Fp levels with the DMF treatment (Figure 7A,B, $p<0.01$ ) and no change in NADH levels (Figure $7 \mathrm{~A}, \mathrm{C})$. The redox ratio $\mathrm{Fp} /(\mathrm{NADH}+\mathrm{Fp}$ ) was significantly lower in the DMFtreated group (Figure 7D, $p<0.05$ ). DMF treatment did not induce significant changes in Fp or NADH levels within the brainstem (Figure $8 \mathrm{~A}-\mathrm{C}$ ), but it was associated with a significantly lower redox ratio, albeit with a small effect size (Figure $8 \mathrm{D}, p<0.05$ ).

\subsection{No Toxic Effect Was Associated with DMF Treatment}

DMF treatment was not associated with changes in the peripheral blood hematologic counts (Figure 9), brain neuronal markers (synaptic proteins, Figure 10), or NFL in the 
brain tissue, plasma, or CSF (Figure 11). One DMF-treated SIV-infected animal developed a B cell lymphoma, commonly observed in immune-deficient SIV-infected macaques.
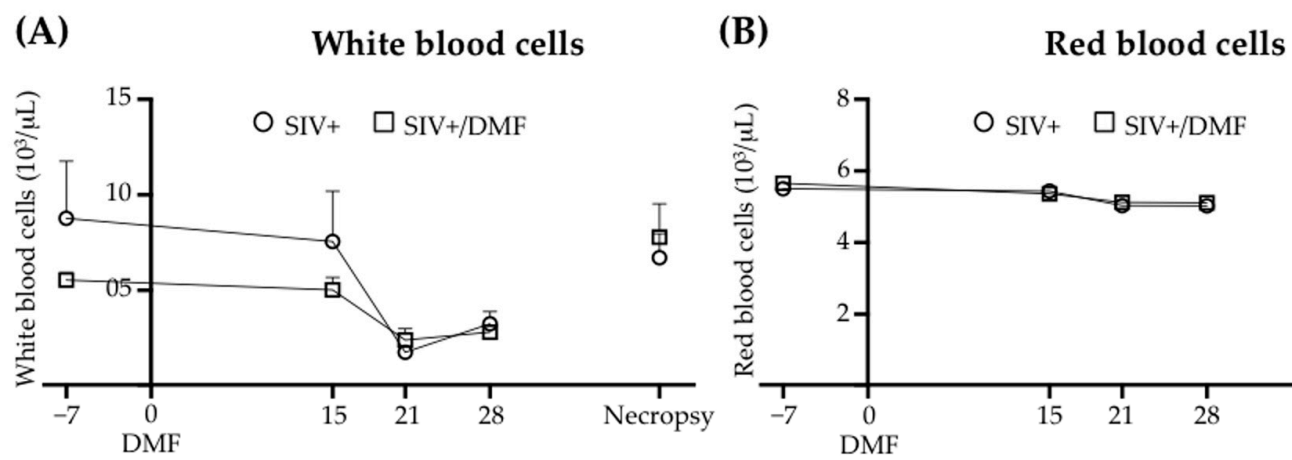

(C)

Hemoglobin
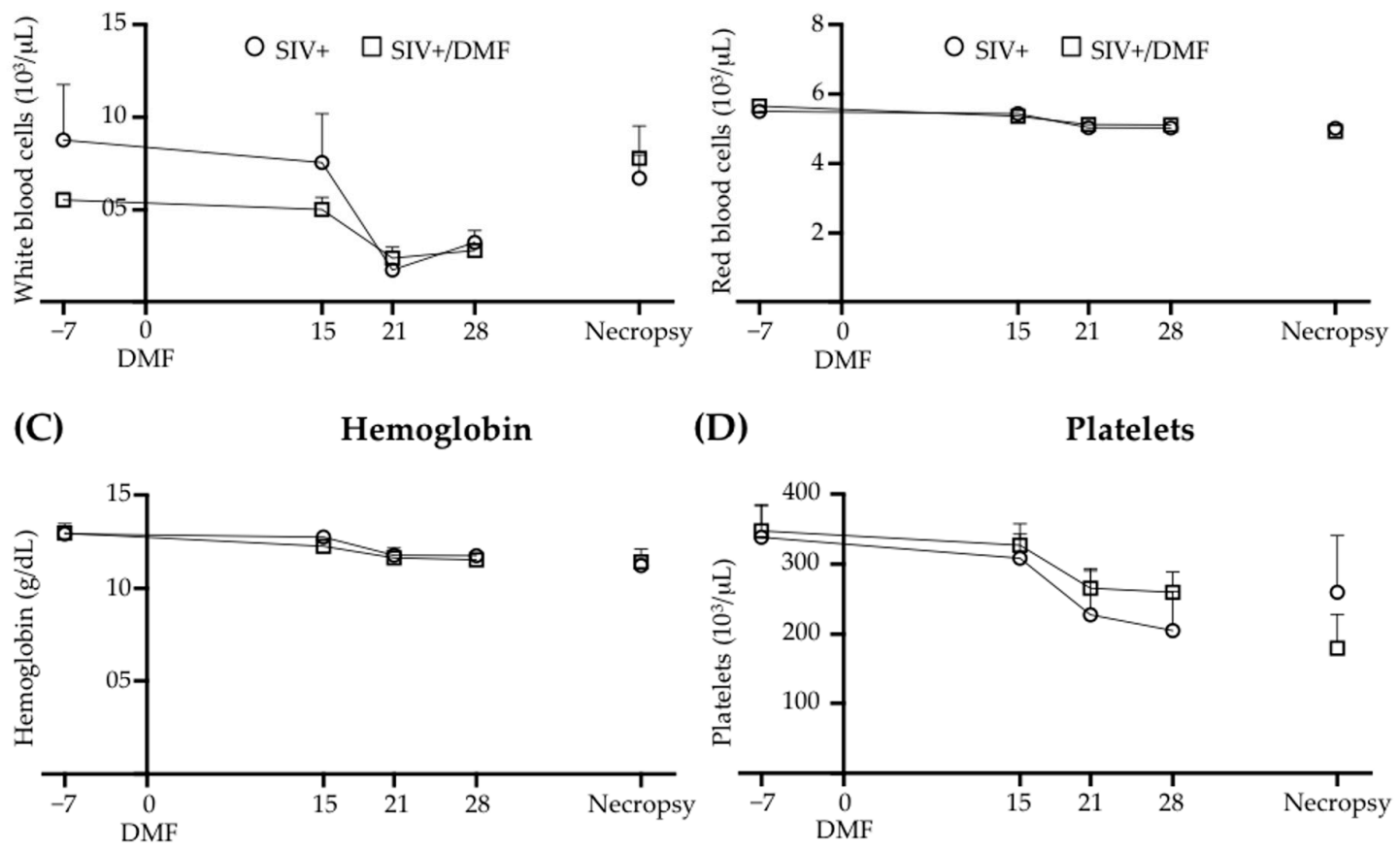

(D)

Platelets

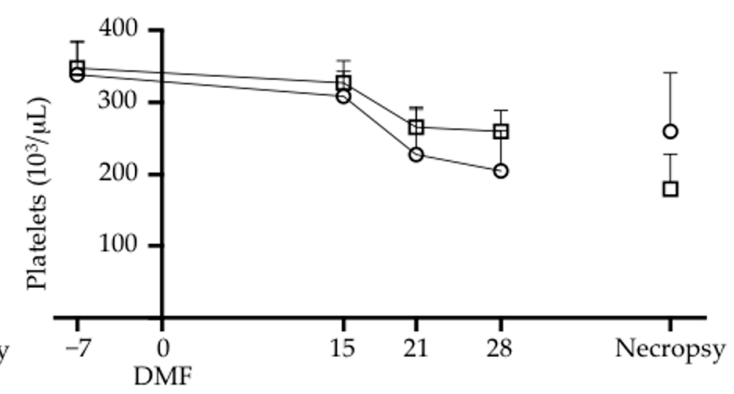

(E)

Hematocrit

(F)

Neutrophils
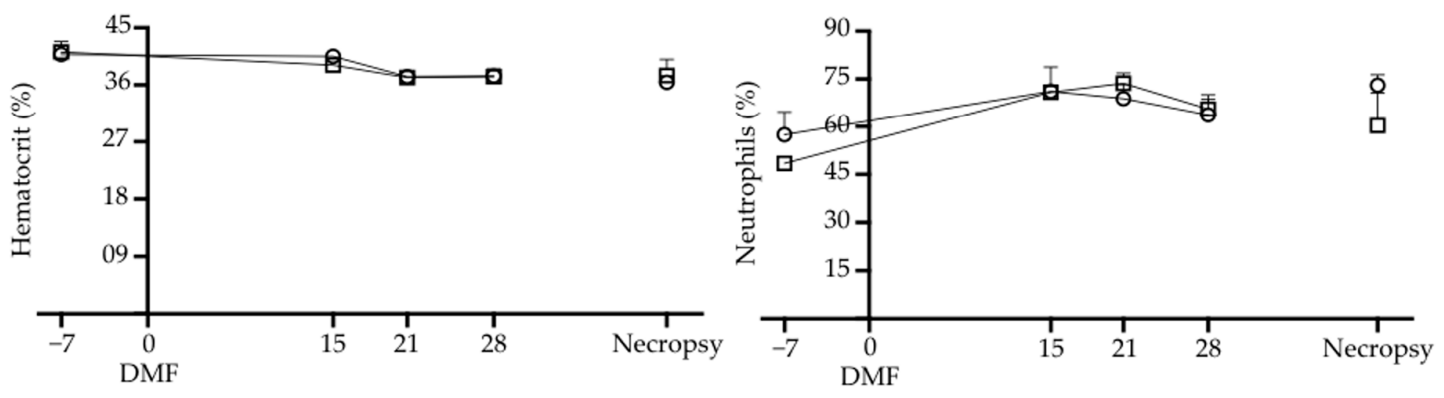

(G)

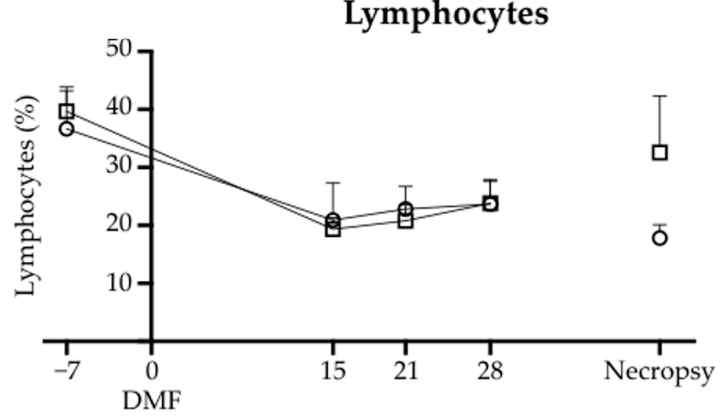

(H)

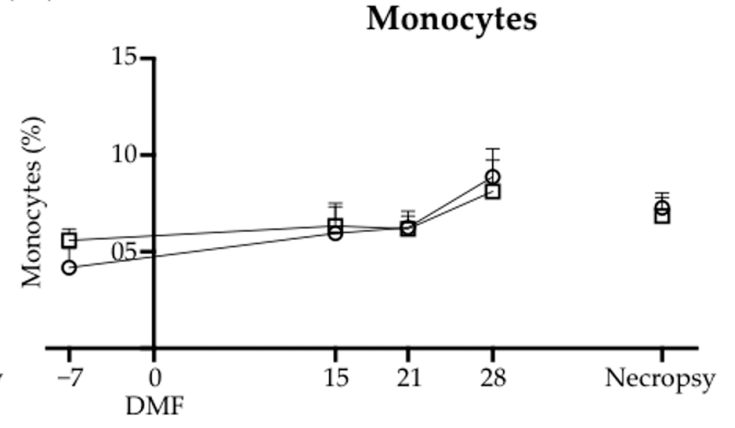

Figure 9. DMF treatment did not associate with changes in blood cell counts. (A) White blood cell count. (B) Red blood cell count. (C) Hemoglobin level. (D) Platelet count. (E) Hematocrit level in total blood. (F) Percent of neutrophils from total white cells. (G) Percent of lymphocytes from total white cells. (H) Percent of monocytes from total white cells. Blood parameters were assessed at time points (days) from the day of initiation of the DMF treatment. Each point represents the average for each treatment group at that time point. Nine SIV-infected rhesus macaques were used in the study (4 untreated animals and 5 DMF-treated (90 mg total DMF daily)). All values are expressed as mean \pm SEM. 
(A)

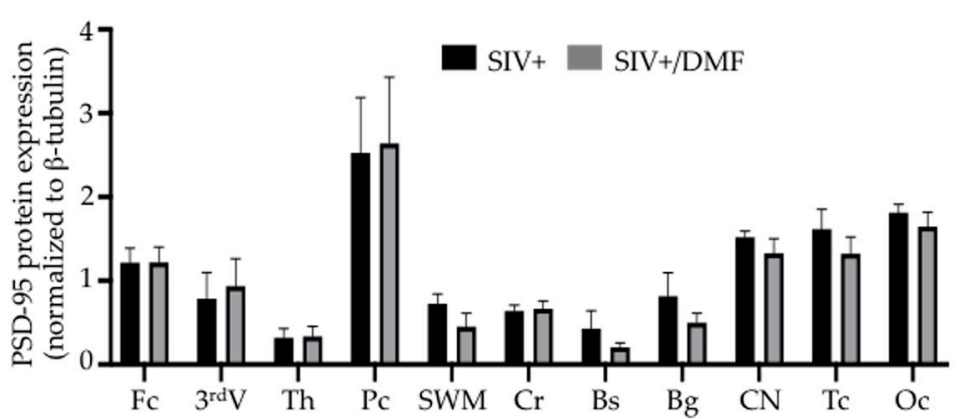

(C)

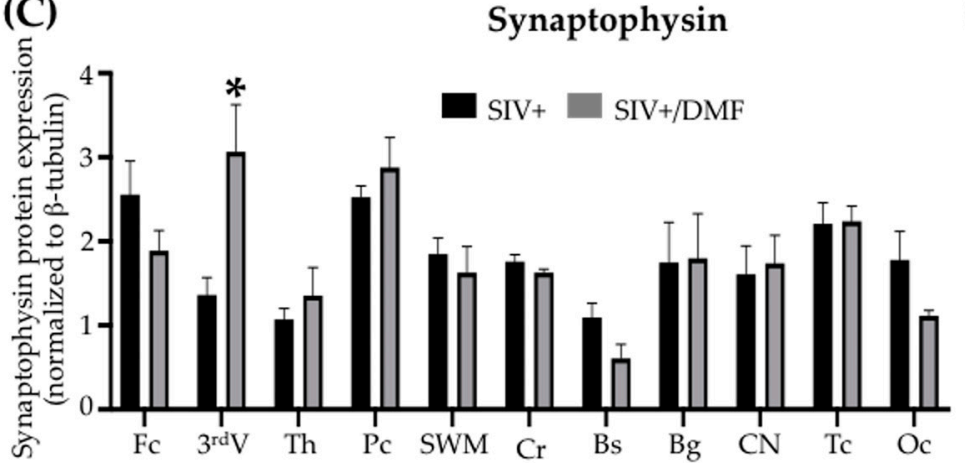

(E)

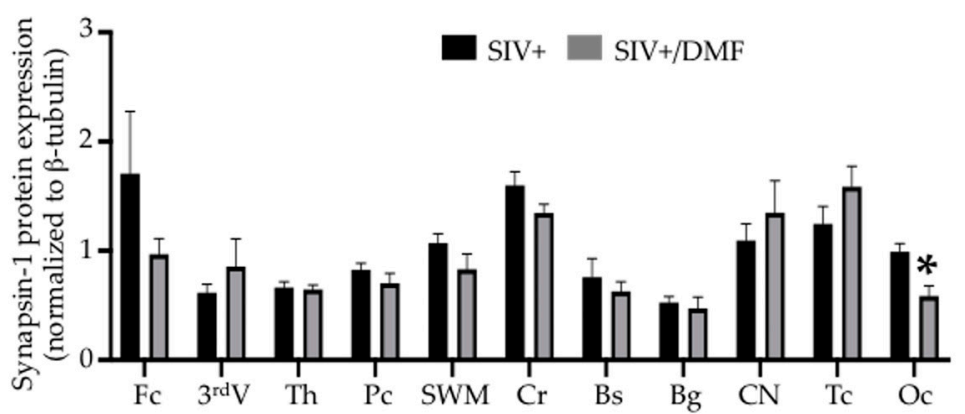

(B)

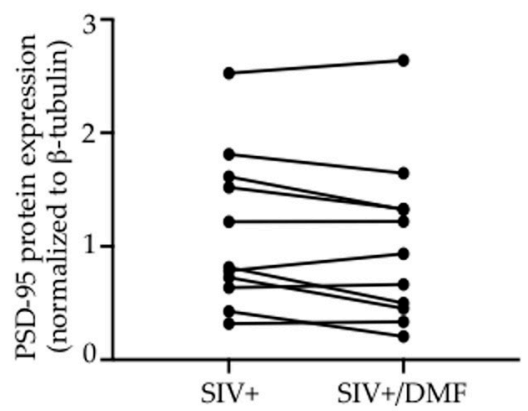

(D)

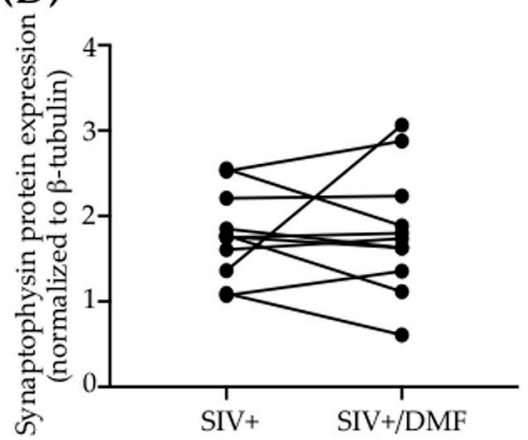

(F)

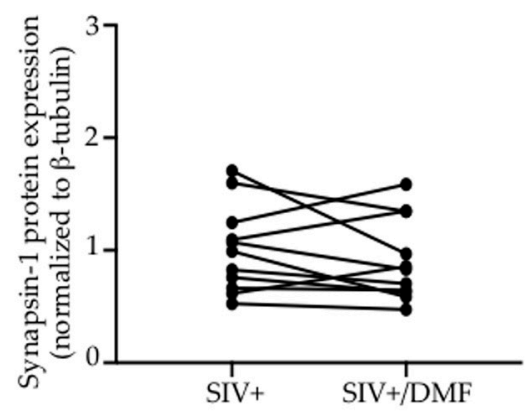

Figure 10. DMF treatment was associated with limited regional changes in expression of the synaptic proteins synaptophysin and synapsin I, but not PSD-95, in the brains of SIV-infected macaques. (A,B) No significant differences in PSD-95 (postsynaptic protein) expression were observed in individual regions or overall in the brains with DMF treatment. (C) A significant increase in synaptophysin (pre-synaptic protein) expression was observed in the third ventricle (Student's unpaired $t$-test, ${ }^{*} p<0.05$ ) but without a difference in the overall mean brain expression (D) with DMF treatment. (E) A significant decrease in synapsin 1 (pre-synaptic protein) expression was observed in the occipital cortex (Student's unpaired $t$-test, $\left.{ }^{*} p<0.05\right)$ but without a difference in the overall mean brain expression (F) with DMF treatment. Eleven brain regions from nine SIV-infected rhesus macaques (4 untreated animals and 5 DMF-treated (90 mg total DMF daily)) were analyzed using Western blotting. In the right panels, each dot represents the mean expression of one brain region from all animals in the group. Fc: frontal cortex, $3^{\text {rd }} \mathrm{V}$ : third ventricle, Th: thalamus, Pc: parietal cortex, SWM: subcortical white matter, Cr: cerebellum, Bs: brainstem, Bg: basal ganglia, CN: caudate nuclei, Tc: temporal cortex, Oc: occipital cortex. All values are expressed as mean \pm SEM. 


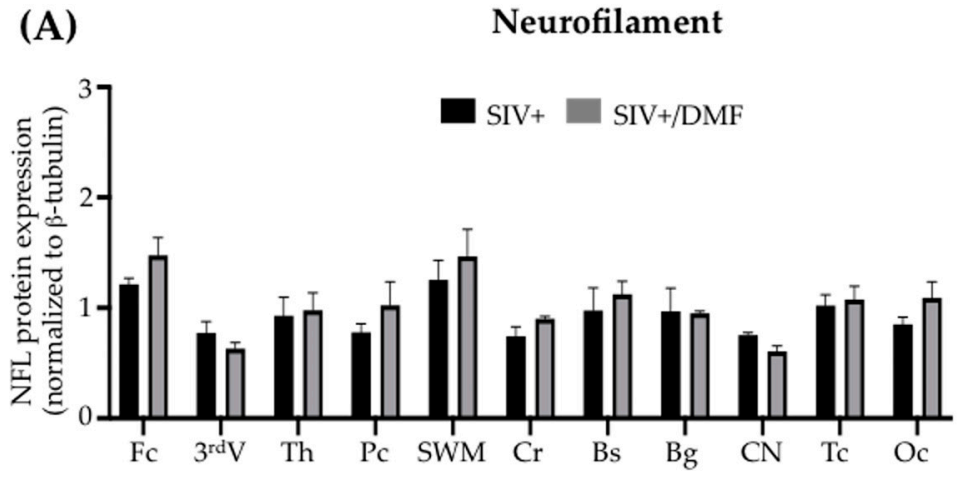

(C)

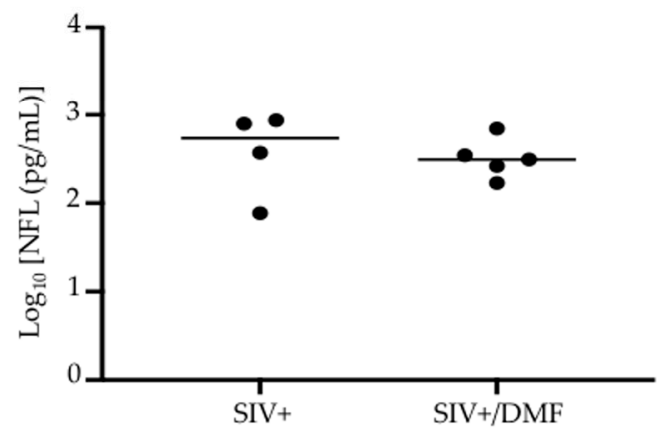

(B)

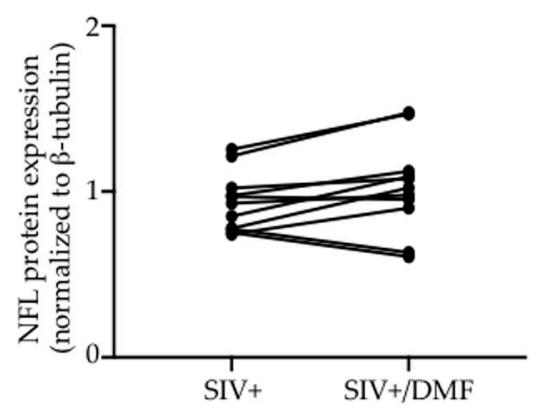

CSF NFL
(D)

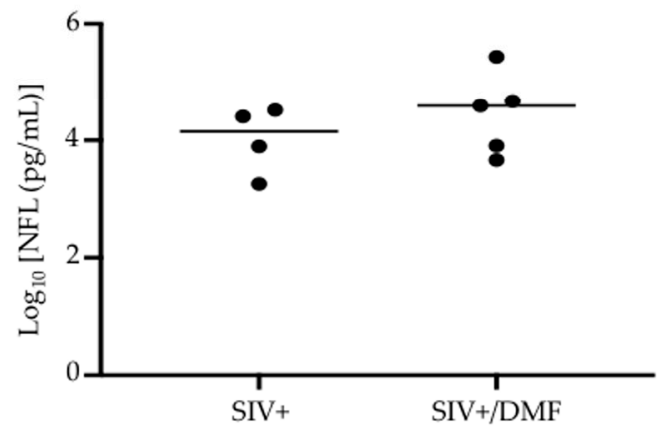

Figure 11. DMF treatment was not associated with changes in NFL. (A,B) NFL in the brain tissue, as determined using Western blot. Eleven brain regions from 9 SIV-infected rhesus macaques (4 untreated animals and 5 DMF-treated (90 mg total DMF daily)) were analyzed. In panel (B), each dot represents the mean average for each region and group. (C) The plasma and (D) cerebrospinal fluid (CSF) NFL did not change with the DMF treatment. NFL was quantified using a Singleplex Assay (Meso Scale Discovery, Rockville, MD, USA). Each dot represents the NFL value for each animal. Fc: frontal cortex, $3^{\text {rd }}$ : third ventricle, Th: thalamus, Pc: parietal cortex, SWM: subcortical white matter, Cr: cerebellum, Bs: brainstem, Bg: basal ganglia, $\mathrm{CN}$ : caudate nuclei, Tc: temporal cortex, Oc: occipital cortex. All values are expressed as mean $\pm \mathrm{SEM}$.

\subsection{DMF Treatment Was Associated with a Higher Expression of VCAM-1 and ICAM-1}

DMF treatment was associated with no changes in the expression of GFAP (glial fibrillary acidic protein) and an increased expression of VCAM-1 $(p<0.01)$ and ICAM-1 (intercellular adhesion molecule 1, $p<0.01$ ) (Figure 12). DMF treatment was not associated with changes in HLA-DR (human leukocyte antigen-DR isotype) or CD68 (Figure 13). 

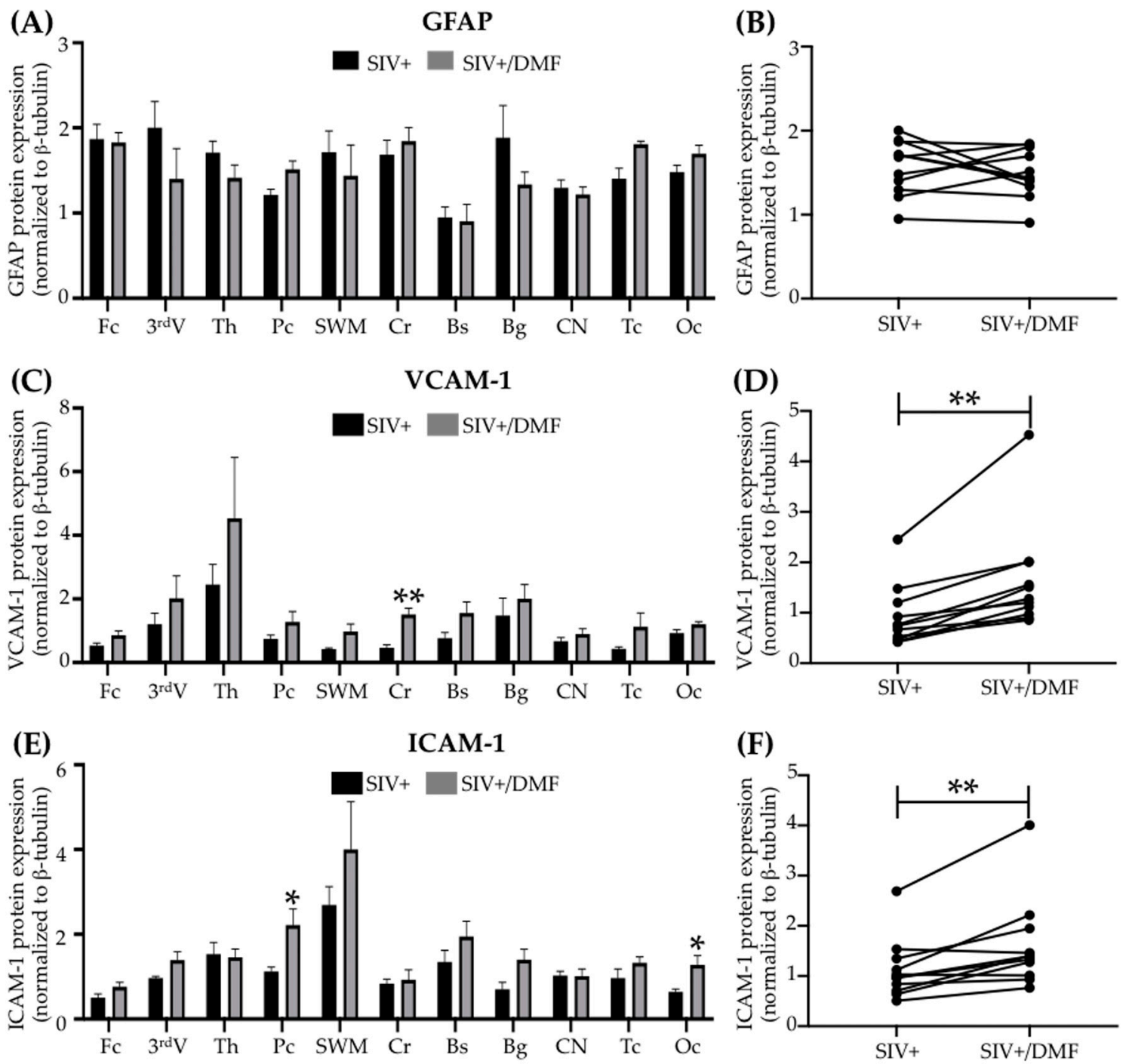

Figure 12. DMF treatment was associated with higher VCAM-1 and ICAM-1 expression in the brains of SIV-infected macaques. (A,B) No significant differences in the GFAP expression were observed in individual regions or overall in the brains with DMF treatment. (C) A significant increase in VCAM-1 expression in the cerebellum (Student's unpaired $t$-test, ${ }^{* *} p<0.01$ ) and in the overall mean expression (D) with DMF treatment (Student's paired $t$-test, ${ }^{* *} p<0.01$ ). (E) A significant increase in the ICAM-1 expression in the parietal and occipital cortexes (Student's unpaired $t$-test, ${ }^{*} p<0.05$ ) and in the overall mean expression (F) with DMF treatment (Student's paired $t$-test, ${ }^{* *} p<0.01$ ). Eleven brain regions from 9 SIV-infected rhesus macaques (4 untreated animals and 5 DMF-treated (90 mg total DMF daily)) were analyzed using Western blotting. In the right panels, each dot represents the mean expression of one brain region from all animals in the group. Fc: frontal cortex, $3^{\text {rd }}$ V: third ventricle, Th: thalamus, Pc: parietal cortex, SWM: subcortical white matter, Cr: cerebellum, Bs: brainstem, Bg: basal ganglia, CN: caudate nuclei, Tc: temporal cortex, Oc: occipital cortex. All values are expressed as mean \pm SEM. 

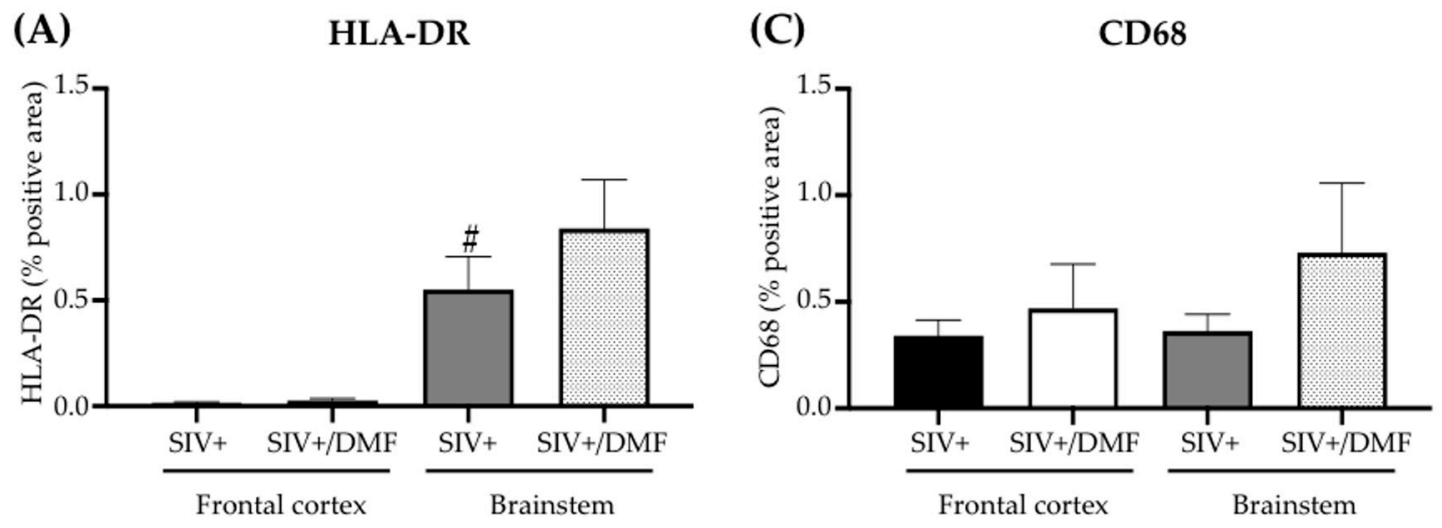

(B)

Frontal cortex

(D)
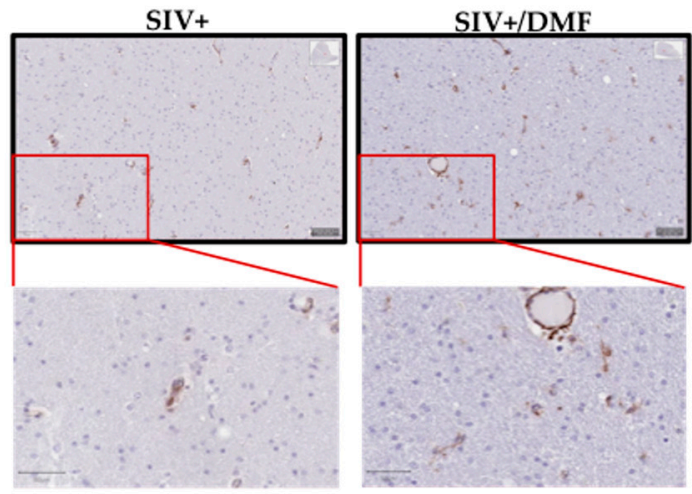

Brainstem
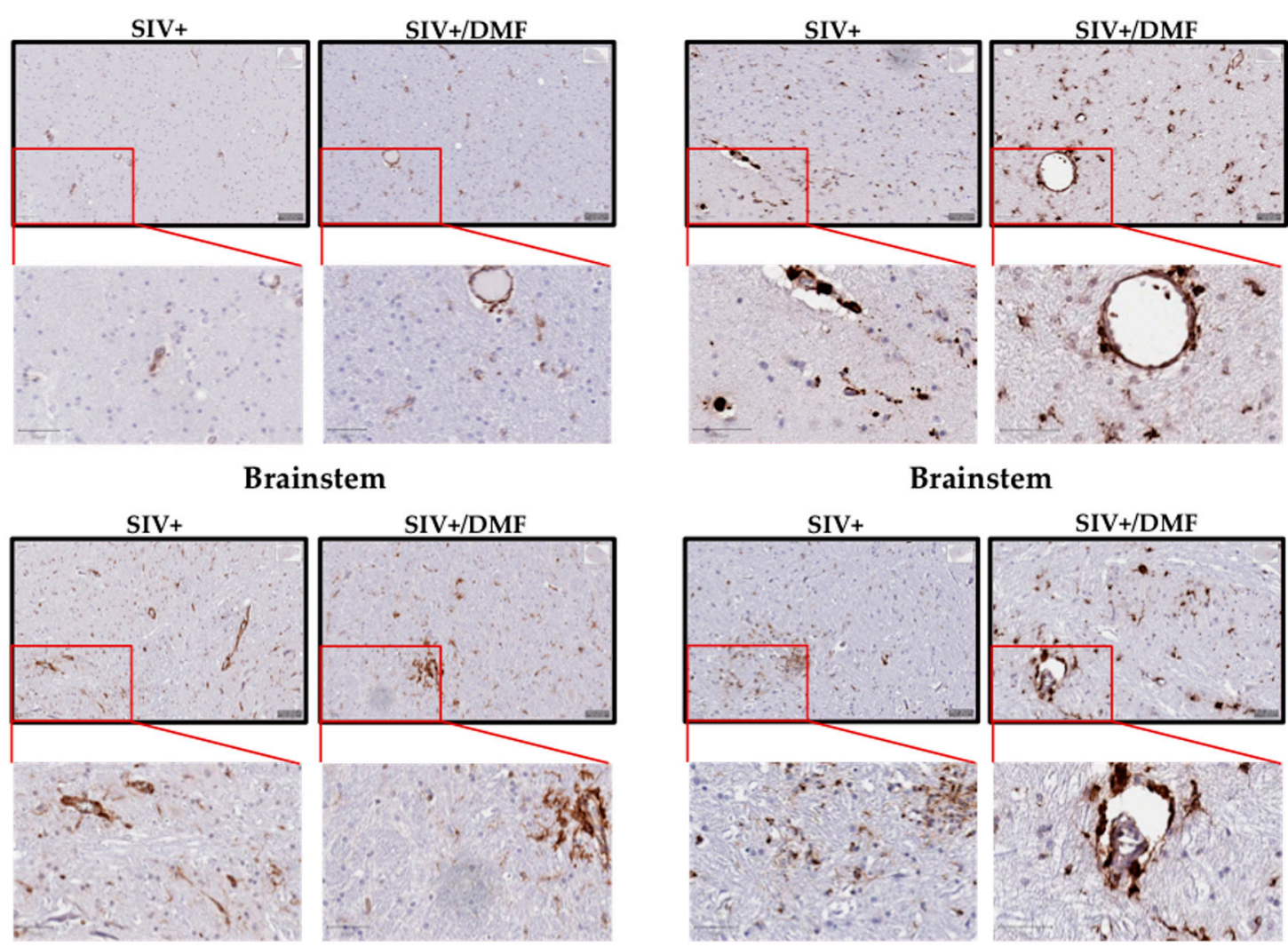

Brainstem

SIV+
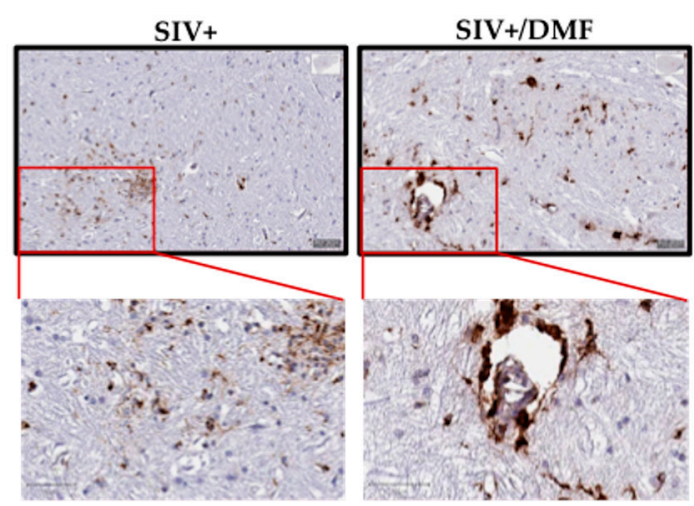

Figure 13. DMF treatment was not associated with the altered brain expression of HLA-DR (monocyte/microglia activation) or CD68 (monocyte marker). (A) Quantification of HLA-DR in the frontal cortex and brainstem. The brainstem showed a higher expression of HLA-DR than the frontal cortex (statistical analysis was done with Student's unpaired $t$-test, ${ }^{\#} p<0.05$ comparing the frontal cortex vs. the brainstem in the SIV+ group) and the DMF treatment showed no effect on the HLADR expression. (B) Representative labeling for HLA-DR in the frontal cortex (top panels) and brainstem (bottom panels). (C) Quantification of CD68 in the frontal cortex and brainstem. The DMF treatment showed no effect on the CD68 expression. (D) Representative labeling for CD68 in the frontal cortex (top panels) and brainstem (bottom panels). Four contiguous sections from each region were harvested from each animal for labeling with each antibody. Nine SIV-infected rhesus macaques were used in the study ( 4 untreated animals and 5 DMF-treated ( $90 \mathrm{mg}$ total DMF daily)). Images from all sections were taken using a digital slide scanner (Aperio AT2, Leica Biosystem, Wetzlar, Germany) at 20× magnification and were analyzed with QuPath software (version 0.2.0-m5), as previously reported [46]. Quantifications are expressed as the mean percent of positive staining from all the stained areas $\pm \mathrm{SEM}$. 


\section{Discussion}

The persistent risk for brain injury and associated neurocognitive impairment in persons living with HIV, despite the suppression of virus replication with antiretroviral therapy, underscores the need for the identification of adjunctive neuroprotective strategies $[66,67]$. Abundant evidence, including data from our studies, suggests that oxidative stress and neuroinflammation contribute to the risk for brain injury in acute and chronic HIV and SIV infections [4,6,20,68-71]. To address the need for identifying an effective adjunctive neuroprotection strategy, we examined the ability of DMF-an FDA-approved, anti-oxidative, and anti-inflammatory drug - to limit brain oxidative stress and neuroinflammation in chronically SIV-infected rhesus macaques, which is the non-human primate model of HIV infection.

To mimic the robust oxidative stress and neuroinflammation that is associated with HIV infection of the brain, we used the immune-deficient ( $\mathrm{CD} 8^{+} \mathrm{T}$ lymphocyte depletion) model of SIV neuropathogenesis, which consistently produces the robust brain inflammation that is associated with SIV replication [39]. We approximated the DMF doses used in humans for the treatment of multiple sclerosis $(\approx 7 \mathrm{mg} / \mathrm{kg}$ total daily dose $)$ through enteral administration in the macaques and confirmed the single-dose kinetics of DMF absorption and metabolism, as indicated by the appearance of therapeutic levels of DMF's biologically active in vivo metabolite, namely, MMF, in the plasma of uninfected animals prior to their entry into the infection arm of the study [72]. We detected the MMF plasma levels $(\approx 4.1 \mu \mathrm{M}) 60$ min after the administration of DMF, followed by reduced levels at 120 and $240 \mathrm{~min}$, which is consistent with prior studies in humans [50-52]. Numerous published studies, including our own, have demonstrated physiological antioxidant and anti-inflammatory responses in cultured cells that were exposed to MMF in this concentration range, thus suggesting that we can indeed achieve therapeutic levels in macaques when dosing with DMF concentrations used in humans [20]. Although we did not determine MMF levels in brain tissue or CSF, DMF treatments in animal models have been shown to achieve therapeutic levels in brain tissue [12,73]. Similar results have been shown by the nanoparticle-mediated oral delivery of formulated DMF in rodents detecting MMF in the brain [74]. Thus, our study contextualized the biological parameters of effective human therapeutic applications of DMF therapy for neuroprotection in the macaque model of HIV neuropathogenesis.

When comparing the DMF-treated vs. untreated SIV-infected animals, we observed the following associations with the DMF treatment: (i) higher brain expression of antioxidant enzymes NQO1, GPX1, and HO-1; (ii) lower brain expression of protein and DNA oxidation markers (3NT (proteins), 8-OHdG (DNA)); (iii) a more reduced redox state in both the frontal cortex and brainstem; (iv) higher spleen expression of antioxidant enzymes PRDX1 and HO-2; (v) no differences in the CSF and plasma NFL levels or SIV viral loads; (vi) no differences in the peripheral blood hematologic counts. Moreover, our analyses included 11 brain regions involving cortical and brainstem anatomical sites, which would identify global brain responses to DMF treatment. These observations suggest that chronic DMF administration at human-equivalent therapeutic doses to SIV-infected, immune-deficient rhesus macaques safely induced robust antioxidant responses throughout the brain.

We saw no evidence for neurotoxic effects, based upon the CSF and plasma NFL levels, although we cannot conclude that DMF demonstrated a protective effect against neuronal injury in these animals. We also found no evidence for anti-inflammatory effects from the DMF treatment in these animals. The lack of evidence in this study for significant neuroprotective or anti-inflammatory effects of DMF could be a consequence of the accelerated systemic and CNS disease progression in this SIV macaque model. SIV-infected macaques exhibit virological, immunological, and clinical manifestations that are similar to HIV-1 infected humans on a shorter time scale of 2 to 3 years. However, in CD8 ${ }^{+}-\mathrm{T}-$ lymphocytedepleted models, the SIV disease timeline is significantly accelerated ( $<6$ months) with increased viremia, neuroinflammation, and CNS pathology, including SIV encephalitis [39]. This more fulminant disease model may obscure the anti-inflammatory and neuroprotective 
effects of DMF. Thus, whether DMF has the potential to prevent neuroinflammation and neuronal injury in SIV / HIV infection remains to be determined and future studies should examine DMF's effects on neuroinflammation and brain injury in immune-competent macaques at early time points prior to significant disease progression.

Our demonstration of higher brain expression of antioxidant enzymes, lower DNA and protein oxidation, and a more reduced redox state in DMF-treated SIV-infected animals suggests a direct link between enzyme induction and reduced oxidative stress and injury. Among the enzymes induced were NQO1, GPX1, HO-1, and PRDX1. NQO1 is important in neuroprotection, particularly within dopaminergic regions, because of its ability to reduce reactive oxygen species (ROS), reduce/detoxify dopamine metabolites, and stabilize p53 [75]. GPX1 is a major detoxifier of peroxides, including lipid peroxides, and it is expressed in the nucleus, cytoplasm, and mitochondria; it is a sensitive and specific suppressor of ROS production and oxidative damage [61]. It is particularly important for mitochondrial $\mathrm{H}_{2} \mathrm{O}_{2}$ scavenging [76]. It has been shown that fumarate interacts directly with GPX1 to induce GPX1 activation [77]. Furthermore, DMF treatment has been shown to increase levels of reduced glutathione (GSH), which reduces peroxynitrite and the associated oxidative injury cellular injury [19]. HO-1 serves a critical function in heme detoxification (a major intra- and extracellular pro-oxidant), with a linkage to neuronal injury, recovery, and neuroprotection [78-81], especially in persons living with HIV $[4,34,36,68,69]$. PRDX1 is present in glial cells, primarily in the nucleus, cytosol, and mitochondria, and it removes peroxides, including $\mathrm{H}_{2} \mathrm{O}_{2}$, and peroxynitrite [82]. It is an effective suppressor of microglial activation $[83,84]$ and it serves protective functions in neurodegenerative diseases [82]. Thus, there are numerous enzymatic pathways that may be activated by DMF treatment that could lead to a state of reduced oxidative stress in SIV infection, and presumably HIV infection.

We speculate that a major effect of DMF treatment is the reduction of mitochondrial oxidative injury. Particularly relevant to this are the nicotinamide adenine dinucleotide redox pair $\left(\mathrm{NAD}^{+} / \mathrm{NADH}\right)$ and the flavin adenine dinucleotide redox pair $\left(\mathrm{FAD} / \mathrm{FADH}_{2}\right)$. $\mathrm{NADH}$ and $\mathrm{FADH}_{2}$ are important electron donors in oxidative processes in the mitochondria $[85,86]$. Both redox pairs have critical roles in regulating the mitochondrial redox state and cellular metabolism [87-90], which generates ROS that can lead to increased oxidative stress when the redox homeostasis is dysregulated [91-93]. The optical redox ratio $\mathrm{Fp} /(\mathrm{NADH}+\mathrm{Fp})$ linearly correlates with the $\mathrm{NAD}^{+} /\left(\mathrm{NADH}+\mathrm{NAD}^{+}\right)$ratio that is determined using liquid chromatography-mass spectrometry and reflects the mitochondrial redox state [94,95]. A higher Fp or lower NADH or higher optical redox ratio corresponds to a higher mitochondrial ROS level; either oxidative or reductive extreme correlates with a higher ROS level $[48,96]$ and a more oxidized redox state (a higher redox ratio) is associated with neurodegeneration $[97,98]$.

\section{Conclusions}

Our results suggest a potential therapeutic benefit of DMF treatment in persons living with HIV, as oxidative stress in the periphery and the CNS are common features of HIV infection $[4,20,36,68,70,71,99-102]$. The DMF-treated macaques used in this study were severely immune-deficient (secondary to $\mathrm{CD} 8^{+} \mathrm{T}$ lymphocyte depletion) with high SIV loads, and DMF treatment showed no apparent adverse effects above those attributable to SIV infection. This suggests its potential safe use in persons living with HIV. Notably, in such groups of people who achieve virus suppression with cART and who subsequently undergo organ transplantation and associated immunosuppressive therapy, survival rates are equivalent to those of people without HIV [103]. We, therefore, have suggested the potential safe use of DMF in persons living with HIV as an adjunctive neuroprotective therapy [34]. Whether DMF prevents neuronal injury in SIV-infected macaques remains to be determined, where this is a future goal for testing DMF effects on early brain injury in SIV infection in immune-competent macaques as a model for neuroprotection in acute HIV infection [6]. 
Supplementary Materials: The following are available online at https:/ / www.mdpi.com/2076-3 921/10/3/416/s1, Figure S1: DMF treatment does not associate with changes in expression of antioxidant enzymes in liver or thymus.

Author Contributions: Conceptualization, D.L.K., P.V. and A.J.G.; methodology, D.L.K., Y.G.-M., R.G., A.L.G. and H.N.X.; formal analysis, H.N.X. and Y.G.-M.; investigation, P.V., R.G., A.L.G., H.N.X. and Y.G.M.; resources, D.J.I., X.A.-H. and C.M.; data curation, D.L.K., H.N.X. and Y.G.-M.; writing-original draft preparation, Y.G.-M.; writing—review and editing, D.L.K., H.N.X., A.J.G., A.L.G., D.J.I. and Y.G.-M.; supervision, D.L.K. and D.J.I.; project administration, D.L.K.; funding acquisition, D.L.K. All authors have read and agreed to the published version of the manuscript.

Funding: This research was funded by the National Institutes of Health (NIH): R01 MH104134-01 (D.L.K.), R01 MH111389-01 (D.L.K.), and R25 GM071745 (R.G.).

Institutional Review Board Statement: This study was performed following the Institutional Animal Care and Use Committee (IACUC) of Tulane University approved protocol, number A3180-01, in accordance with the Animal Welfare Act and other federal statutes and regulations relating to animals in United States of America. This animal protocol was approved by the Tulane University's Animal Care and Use Committee on 21 August 2017.

Informed Consent Statement: Not applicable.

Data Availability Statement: The data presented in this study are available within the article and in supplementary material.

Acknowledgments: We thank the support from the Cell and Developmental Biology (CDB) Microscopy Core, Perelman School of Medicine, University of Pennsylvania.

Conflicts of Interest: The authors declare no conflict of interest. The funders had no role in the design of the study; in the collection, analyses, or interpretation of data; in the writing of the manuscript, or in the decision to publish the results.

\section{References}

1. Brenchley, J.M.; Price, D.A.; Schacker, T.W.; Asher, T.E.; Silvestri, G.; Rao, S.; Kazzaz, Z.; Bornstein, E.; Lambotte, O.; Altmann, D.; et al. Microbial translocation is a cause of systemic immune activation in chronic HIV infection. Nat. Med. 2006, 12, 1365-1371. [CrossRef]

2. Ancuta, P.; Kamat, A.; Kunstman, K.J.; Kim, E.Y.; Autissier, P.; Wurcel, A.; Zaman, T.; Stone, D.; Mefford, M.; Morgello, S.; et al. Microbial translocation is associated with increased monocyte activation and dementia in AIDS patients. PLoS ONE 2008, 3, e2516. [CrossRef] [PubMed]

3. Eden, A.; Price, R.W.; Spudich, S.; Fuchs, D.; Hagberg, L.; Gisslen, M. Immune activation of the central nervous system is still present after $>4$ years of effective highly active antiretroviral therapy. J. Infect. Dis. 2007, 196, 1779-1783. [CrossRef]

4. Gill, A.J.; Kovacsics, C.E.; Cross, S.A.; Vance, P.J.; Kolson, L.L.; Jordan-Sciutto, K.L.; Gelman, B.B.; Kolson, D.L. Heme oxygenase-1 deficiency accompanies neuropathogenesis of HIV-associated neurocognitive disorders. J. Clin. Investig. 2014, 124, 4459-4472. [CrossRef] [PubMed]

5. Ivanov, A.V.; Valuev-Elliston, V.T.; Ivanova, O.N.; Kochetkov, S.N.; Starodubova, E.S.; Bartosch, B.; Isaguliants, M.G. Oxidative Stress during HIV Infection: Mechanisms and Consequences. Oxid. Med. Cell. Longev. 2016, 2016, 8910396. [CrossRef] [PubMed]

6. Garcia-Mesa, Y.; Garza, R.; Diaz Ortiz, M.E.; Gruenewald, A.L.; Bastien, B.L.; Lobrovich, R.; Irwin, D.J.; Betts, M.R.; Silvestri, G.; Kolson, D.L. Regional Brain Recovery from Acute Synaptic Injury in Simian Immunodeficiency Virus-Infected Rhesus Macaques Associates with Heme Oxygenase Isoform Expression. J. Virol. 2020, 94, e01102-20. [CrossRef]

7. Fuller, R.A.; Westmoreland, S.V.; Ratai, E.; Greco, J.B.; Kim, J.P.; Lentz, M.R.; He, J.; Sehgal, P.K.; Masliah, E.; Halpern, E.; et al. A prospective longitudinal in vivo $1 \mathrm{H}$ MR spectroscopy study of the SIV/macaque model of neuroAIDS. BMC Neurosci. 2004, 5, 10. [CrossRef]

8. Brenchley, J.M.; Price, D.A.; Douek, D.C. HIV disease: Fallout from a mucosal catastrophe? Nat. Immunol. 2006, 7, 235-239. [CrossRef]

9. Brenchley, J.M.; Douek, D.C. HIV infection and the gastrointestinal immune system. Mucosal. Immunol. 2008, 1, 23-30. [CrossRef]

10. Zhang, M.; An, C.; Gao, Y.; Leak, R.K.; Chen, J.; Zhang, F. Emerging roles of Nrf2 and phase II antioxidant enzymes in neuroprotection. Prog. Neurobiol. 2013, 100, 30-47. [CrossRef]

11. Ellrichmann, G.; Petrasch-Parwez, E.; Lee, D.H.; Reick, C.; Arning, L.; Saft, C.; Gold, R.; Linker, R.A. Efficacy of fumaric acid esters in the R6/2 and YAC128 models of Huntington's disease. PLoS ONE 2011, 6, e16172. [CrossRef] [PubMed]

12. Linker, R.A.; Lee, D.H.; Ryan, S.; van Dam, A.M.; Conrad, R.; Bista, P.; Zeng, W.; Hronowsky, X.; Buko, A.; Chollate, S.; et al. Fumaric acid esters exert neuroprotective effects in neuroinflammation via activation of the Nrf2 antioxidant pathway. Brain 2011, 134, 678-692. [CrossRef] [PubMed] 
13. Iniaghe, L.O.; Krafft, P.R.; Klebe, D.W.; Omogbai, E.K.I.; Zhang, J.H.; Tang, J. Dimethyl fumarate confers neuroprotection by casein kinase 2 phosphorylation of Nrf2 in murine intracerebral hemorrhage. Neurobiol. Dis. 2015, 82, 349-358. [CrossRef]

14. Jing, X.; Shi, H.; Zhang, C.; Ren, M.; Han, M.; Wei, X.; Zhang, X.; Lou, H. Dimethyl fumarate attenuates 6-OHDA-induced neurotoxicity in SH-SY5Y cells and in animal model of Parkinson's disease by enhancing Nrf2 activity. Neuroscience 2015, 286, 131-140. [CrossRef]

15. Ahuja, M.; Ammal Kaidery, N.; Yang, L.; Calingasan, N.; Smirnova, N.; Gaisin, A.; Gaisina, I.N.; Gazaryan, I.; Hushpulian, D.M.; Kaddour-Djebbar, I.; et al. Distinct Nrf2 Signaling Mechanisms of Fumaric Acid Esters and Their Role in Neuroprotection against 1-Methyl-4-Phenyl-1,2,3,6-Tetrahydropyridine-Induced Experimental Parkinson's-Like Disease. J. Neurosci. 2016, 36, 6332-6351. [CrossRef]

16. Calkins, M.J.; Johnson, D.A.; Townsend, J.A.; Vargas, M.R.; Dowell, J.A.; Williamson, T.P.; Kraft, A.D.; Lee, J.M.; Li, J.; Johnson, J.A The Nrf2/ARE pathway as a potential therapeutic target in neurodegenerative disease. Antioxid. Redox Signal. 2009, 11, 497-508. [CrossRef]

17. Calabrese, V.; Cornelius, C.; Dinkova-Kostova, A.T.; Calabrese, E.J.; Mattson, M.P. Cellular stress responses, the hormesis paradigm, and vitagenes: Novel targets for therapeutic intervention in neurodegenerative disorders. Antioxid. Redox Signal. 2010, 13, 1763-1811. [CrossRef] [PubMed]

18. Dinkova-Kostova, A.T.; Baird, L.; Holmstrom, K.M.; Meyer, C.J.; Abramov, A.Y. The spatiotemporal regulation of the Keap1-Nrf2 pathway and its importance in cellular bioenergetics. Biochem. Soc. Trans. 2015, 43, 602-610. [CrossRef]

19. Scannevin, R.H.; Chollate, S.; Jung, M.Y.; Shackett, M.; Patel, H.; Bista, P.; Zeng, W.; Ryan, S.; Yamamoto, M.; Lukashev, M.; et al. Fumarates promote cytoprotection of central nervous system cells against oxidative stress via the nuclear factor (erythroid-derived 2)-like 2 pathway. J. Pharmacol. Exp. Ther. 2012, 341, 274-284. [CrossRef]

20. Cross, S.A.; Cook, D.R.; Chi, A.W.; Vance, P.J.; Kolson, L.L.; Wong, B.J.; Jordan-Sciutto, K.L.; Kolson, D.L. Dimethyl fumarate, an immune modulator and inducer of the antioxidant response, suppresses HIV replication and macrophage-mediated neurotoxicity: A novel candidate for HIV neuroprotection. J. Immunol. 2011, 187, 5015-5025. [CrossRef]

21. Wilms, H.; Sievers, J.; Rickert, U.; Rostami-Yazdi, M.; Mrowietz, U.; Lucius, R. Dimethylfumarate inhibits microglial and astrocytic inflammation by suppressing the synthesis of nitric oxide, IL-1beta, TNF-alpha and IL-6 in an in-vitro model of brain inflammation. J. Neuroinflamm. 2010, 7, 30. [CrossRef]

22. Gillard, G.O.; Collette, B.; Anderson, J.; Chao, J.; Scannevin, R.H.; Huss, D.J.; Fontenot, J.D. DMF, but not other fumarates, inhibits NF-kappaB activity in vitro in an Nrf2-independent manner. J. Neuroimmunol. 2015, 283, 74-85. [CrossRef]

23. Loewe, R.; Holnthoner, W.; Groger, M.; Pillinger, M.; Gruber, F.; Mechtcheriakova, D.; Hofer, E.; Wolff, K.; Petzelbauer, P. Dimethylfumarate inhibits TNF-induced nuclear entry of NF-kappa B/p65 in human endothelial cells. J. Immunol. 2002, 168, 4781-4787. [CrossRef] [PubMed]

24. Nicolay, J.P.; Muller-Decker, K.; Schroeder, A.; Brechmann, M.; Mobs, M.; Geraud, C.; Assaf, C.; Goerdt, S.; Krammer, P.H.; Gulow, K. Dimethyl fumarate restores apoptosis sensitivity and inhibits tumor growth and metastasis in CTCL by targeting NF-kappaB. Blood 2016, 128, 805-815. [CrossRef]

25. Breuer, J.; Herich, S.; Schneider-Hohendorf, T.; Chasan, A.I.; Wettschureck, N.; Gross, C.C.; Loser, K.; Zarbock, A.; Roth, J.; Klotz, L.; et al. Dual action by fumaric acid esters synergistically reduces adhesion to human endothelium. Mult. Scler. 2018, 24, 1871-1882. [CrossRef]

26. Rubant, S.A.; Ludwig, R.J.; Diehl, S.; Hardt, K.; Kaufmann, R.; Pfeilschifter, J.M.; Boehncke, W.H. Dimethylfumarate reduces leukocyte rolling in vivo through modulation of adhesion molecule expression. J. Investig. Dermatol. 2008, 128, 326-331. [CrossRef] [PubMed]

27. Schilling, S.; Goelz, S.; Linker, R.; Luehder, F.; Gold, R. Fumaric acid esters are effective in chronic experimental autoimmune encephalomyelitis and suppress macrophage infiltration. Clin. Exp. Immunol. 2006, 145, 101-107. [CrossRef]

28. Demir, S.; Heckers, S.; Pedreiturria, X.; Hess, D.; Trampe, A.K.; Chan, A.; Gold, R. Low dose fumaric acid esters are effective in a mouse model of spontaneous chronic encephalomyelitis. J. Neuroimmunol. 2015, 285, 16-21. [CrossRef] [PubMed]

29. Paraiso, H.C.; Kuo, P.C.; Curfman, E.T.; Moon, H.J.; Sweazey, R.D.; Yen, J.H.; Chang, F.L.; Yu, I.C. Dimethyl fumarate attenuates reactive microglia and long-term memory deficits following systemic immune challenge. J. Neuroinflamm. 2018, 15, 100. [CrossRef]

30. Naismith, R.T.; Wolinsky, J.S.; Wundes, A.; LaGanke, C.; Arnold, D.L.; Obradovic, D.; Freedman, M.S.; Gudesblatt, M.; Ziemssen, T.; Kandinov, B.; et al. Diroximel fumarate (DRF) in patients with relapsing-remitting multiple sclerosis: Interim safety and efficacy results from the phase 3 EVOLVE-MS-1 study. Mult. Scler. 2020, 26, 1729-1739. [CrossRef]

31. Derfuss, T.; Mehling, M.; Papadopoulou, A.; Bar-Or, A.; Cohen, J.A.; Kappos, L. Advances in oral immunomodulating therapies in relapsing multiple sclerosis. Lancet Neurol. 2020, 19, 336-347. [CrossRef]

32. Kornberg, M.D.; Bhargava, P.; Kim, P.M.; Putluri, V.; Snowman, A.M.; Putluri, N.; Calabresi, P.A.; Snyder, S.H. Dimethyl fumarate targets GAPDH and aerobic glycolysis to modulate immunity. Science 2018, 360, 449-453. [CrossRef]

33. Grzegorzewska, A.P.; Seta, F.; Han, R.; Czajka, C.A.; Makino, K.; Stawski, L.; Isenberg, J.S.; Browning, J.L.; Trojanowska, M. Dimethyl Fumarate ameliorates pulmonary arterial hypertension and lung fibrosis by targeting multiple pathways. Sci. Rep. 2017, 7, 41605. [CrossRef] [PubMed]

34. Gill, A.J.; Kolson, D.L. Dimethyl fumarate modulation of immune and antioxidant responses: Application to HIV therapy. Crit. Rev. Immunol. 2013, 33, 307-359. [CrossRef] [PubMed] 
35. Ambrosius, B.; Gold, R.; Chan, A.; Faissner, S. Antineuroinflammatory drugs in HIV-associated neurocognitive disorders as potential therapy. Neurol. Neuroimmunol. Neuroinflamm. 2019, 6, e551. [CrossRef]

36. Gill, A.J.; Kovacsics, C.E.; Vance, P.J.; Collman, R.G.; Kolson, D.L. Induction of Heme Oxygenase-1 Deficiency and Associated Glutamate-Mediated Neurotoxicity Is a Highly Conserved HIV Phenotype of Chronic Macrophage Infection That Is Resistant to Antiretroviral Therapy. J. Virol. 2015, 89, 10656-10667. [CrossRef]

37. Scuderi, S.A.; Ardizzone, A.; Paterniti, I.; Esposito, E.; Campolo, M. Antioxidant and Anti-inflammatory Effect of Nrf2 Inducer Dimethyl Fumarate in Neurodegenerative Diseases. Antioxidants 2020, 9, 630. [CrossRef] [PubMed]

38. Brennan, M.S.; Patel, H.; Allaire, N.; Thai, A.; Cullen, P.; Ryan, S.; Lukashev, M.; Bista, P.; Huang, R.; Rhodes, K.J.; et al. Pharmacodynamics of Dimethyl Fumarate Are Tissue Specific and Involve NRF2-Dependent and -Independent Mechanisms. Antioxid. Redox Signal. 2016, 24, 1058-1071. [CrossRef]

39. Williams, K.; Lackner, A.; Mallard, J. Non-human primate models of SIV infection and CNS neuropathology. Curr. Opin. Virol. 2016, 19, 92-98. [CrossRef] [PubMed]

40. Walker, J.A.; Miller, A.D.; Burdo, T.H.; McGrath, M.S.; Williams, K.C. Direct Targeting of Macrophages With Methylglyoxal-BisGuanylhydrazone Decreases SIV-Associated Cardiovascular Inflammation and Pathology. J. Acquir. Immune Defic. Syndr. 2017, 74, 583-592. [CrossRef]

41. Nissim, I.; Horyn, O.; Nissim, I.; Daikhin, Y.; Wehrli, S.L.; Yudkoff, M.; Matschinsky, F.M. Effects of a glucokinase activator on hepatic intermediary metabolism: Study with 13C-isotopomer-based metabolomics. Biochem. J. 2012, 444, 537-551. [CrossRef] [PubMed]

42. Liu, J.; Cui, J.Y.; Lu, Y.F.; Corton, J.C.; Klaassen, C.D. Sex-, Age-, and Race/Ethnicity-Dependent Variations in Drug-Processing and NRF2-Regulated Genes in Human Livers. Drug Metab. Dispos. 2021, 49, 111-119. [CrossRef] [PubMed]

43. Hosick, P.A.; Weeks, M.F.; Hankins, M.W.; Moore, K.H.; Stec, D.E. Sex-Dependent Effects of HO-1 Deletion from Adipocytes in Mice. Int. J. Mol. Sci. 2017, 18, 611. [CrossRef]

44. Westmoreland, S.V.; Halpern, E.; Lackner, A.A. Simian immunodeficiency virus encephalitis in rhesus macaques is associated with rapid disease progression. J. Neurovirol. 1998, 4, 260-268. [CrossRef] [PubMed]

45. Cline, A.N.; Bess, J.W.; Piatak, M., Jr.; Lifson, J.D. Highly sensitive SIV plasma viral load assay: Practical considerations, realistic performance expectations, and application to reverse engineering of vaccines for AIDS. J. Med. Primatol. 2005, 34, 303-312. [CrossRef] [PubMed]

46. Ohm, D.T.; Peterson, C.; Lobrovich, R.; Cousins, K.A.Q.; Gibbons, G.S.; McMillan, C.T.; Wolk, D.A.; Van Deerlin, V.; Elman, L.; Spindler, M.; et al. Degeneration of the locus coeruleus is a common feature of tauopathies and distinct from TDP-43 proteinopathies in the frontotemporal lobar degeneration spectrum. Acta Neuropathol. 2020, 140, 675-693. [CrossRef] [PubMed]

47. Xu, H.N.; Zhao, H.; Chellappa, K.; Davis, J.G.; Nioka, S.; Baur, J.A.; Li, L.Z. Optical Redox Imaging of Fixed Unstained Muscle Slides Reveals Useful Biological Information. Mol. Imaging Biol. 2019, 21, 417-425. [CrossRef]

48. Xu, H.N.; Lin, Z.; Gandhi, C.K.; Amatya, S.; Wang, Y.; Li, L.Z.; Floros, J. Sex and SP-A2 Dependent NAD(H) Redox Alterations in Mouse Alveolar Macrophages in Response to Ozone Exposure: Potential Implications for COVID-19. Antioxidants 2020, 9 , 915. [CrossRef]

49. Xu, H.N.; Li, L.Z. Quantitative redox imaging biomarkers for studying tissue metabolic state and its heterogeneity. J. Innov. Opt. Health Sci. 2014, 7. [CrossRef]

50. Sheikh, S.I.; Nestorov, I.; Russell, H.; O'Gorman, J.; Huang, R.; Milne, G.L.; Scannevin, R.H.; Novas, M.; Dawson, K.T. Tolerability and pharmacokinetics of delayed-release dimethyl fumarate administered with and without aspirin in healthy volunteers. Clin. Ther. 2013, 35, 1582-1594 e1589. [CrossRef] [PubMed]

51. Litjens, N.H.; Burggraaf, J.; van Strijen, E.; van Gulpen, C.; Mattie, H.; Schoemaker, R.C.; van Dissel, J.T.; Thio, H.B.; Nibbering, P.H. Pharmacokinetics of oral fumarates in healthy subjects. Br. J. Clin. Pharmacol. 2004, 58, 429-432. [CrossRef] [PubMed]

52. Litjens, N.H.; van Strijen, E.; van Gulpen, C.; Mattie, H.; van Dissel, J.T.; Thio, H.B.; Nibbering, P.H. In vitro pharmacokinetics of anti-psoriatic fumaric acid esters. BMC Pharmacol. 2004, 4, 22. [CrossRef]

53. Zink, M.C.; Suryanarayana, K.; Mankowski, J.L.; Shen, A.; Piatak, M., Jr.; Spelman, J.P.; Carter, D.L.; Adams, R.J.; Lifson, J.D.; Clements, J.E. High viral load in the cerebrospinal fluid and brain correlates with severity of simian immunodeficiency virus encephalitis. J. Virol. 1999, 73, 10480-10488. [CrossRef]

54. Itoh, K.; Wakabayashi, N.; Katoh, Y.; Ishii, T.; O'Connor, T.; Yamamoto, M. Keap1 regulates both cytoplasmic-nuclear shuttling and degradation of Nrf2 in response to electrophiles. Genes Cells 2003, 8, 379-391. [CrossRef] [PubMed]

55. Itoh, K.; Tong, K.I.; Yamamoto, M. Molecular mechanism activating Nrf2-Keap1 pathway in regulation of adaptive response to electrophiles. Free Radic. Biol. Med. 2004, 36, 1208-1213. [CrossRef]

56. Lee, J.M.; Calkins, M.J.; Chan, K.; Kan, Y.W.; Johnson, J.A. Identification of the NF-E2-related factor-2-dependent genes conferring protection against oxidative stress in primary cortical astrocytes using oligonucleotide microarray analysis. J. Biol. Chem. 2003, 278, 12029-12038. [CrossRef]

57. Kobayashi, M.; Yamamoto, M. Nrf2-Keap1 regulation of cellular defense mechanisms against electrophiles and reactive oxygen species. Adv. Enzyme Regul. 2006, 46, 113-140. [CrossRef] [PubMed]

58. Fourtounis, J.; Wang, I.M.; Mathieu, M.C.; Claveau, D.; Loo, T.; Jackson, A.L.; Peters, M.A.; Therien, A.G.; Boie, Y.; Crackower, M.A. Gene expression profiling following NRF2 and KEAP1 siRNA knockdown in human lung fibroblasts identifies CCL11/Eotaxin-1 as a novel NRF2 regulated gene. Respir. Res. 2012, 13, 92. [CrossRef] 
59. Rosito, M.; Testi, C.; Parisi, G.; Cortese, B.; Baiocco, P.; Di Angelantonio, S. Exploring the Use of Dimethyl Fumarate as Microglia Modulator for Neurodegenerative Diseases Treatment. Antioxidants 2020, 9, 700. [CrossRef]

60. Bloom, D.A.; Jaiswal, A.K. Phosphorylation of Nrf2 at Ser40 by protein kinase C in response to antioxidants leads to the release of Nrf2 from INrf2, but is not required for Nrf2 stabilization/accumulation in the nucleus and transcriptional activation of antioxidant response element-mediated NAD $(\mathrm{P}) \mathrm{H}$ :quinone oxidoreductase-1 gene expression. J. Biol. Chem. 2003, 278, 44675-44682. [CrossRef] [PubMed]

61. Huang, J.Q.; Zhou, J.C.; Wu, Y.Y.; Ren, F.Z.; Lei, X.G. Role of glutathione peroxidase 1 in glucose and lipid metabolism-related diseases. Free Radic. Biol. Med. 2018, 127, 108-115. [CrossRef] [PubMed]

62. Lee, Y.J. Knockout Mouse Models for Peroxiredoxins. Antioxidants 2020, 9, 182. [CrossRef]

63. Alam, J.; Stewart, D.; Touchard, C.; Boinapally, S.; Choi, A.M.; Cook, J.L. Nrf2, a Cap'n'Collar transcription factor, regulates induction of the heme oxygenase-1 gene. J. Biol. Chem. 1999, 274, 26071-26078. [CrossRef]

64. Heikal, A.A. Intracellular coenzymes as natural biomarkers for metabolic activities and mitochondrial anomalies. Biomark. Med. 2010, 4, 241-263. [CrossRef]

65. Georgakoudi, I.; Quinn, K.P. Optical imaging using endogenous contrast to assess metabolic state. Annu. Rev. Biomed. Eng. 2012, 14, 351-367. [CrossRef]

66. Abdelkader, N.F.; Abd El-Latif, A.M.; Khattab, M.M. Telmisartan/17beta-estradiol mitigated cognitive deficit in an ovariectomized rat model of Alzheimer's disease: Modulation of ACE1/ACE2 and AT1/AT2 ratio. Life Sci. 2020, 245, 117388. [CrossRef]

67. Pushpakom, S.; Kolamunnage-Dona, R.; Taylor, C.; Foster, T.; Spowart, C.; Garcia-Finana, M.; Kemp, G.J.; Jaki, T.; Khoo, S.; Williamson, P.; et al. TAILoR (TelmisArtan and InsuLin Resistance in Human Immunodeficiency Virus [HIV]): An Adaptivedesign, Dose-ranging Phase IIb Randomized Trial of Telmisartan for the Reduction of Insulin Resistance in HIV-positive Individuals on Combination Antiretroviral Therapy. Clin. Infect. Dis. 2020, 70, 2062-2072. [CrossRef]

68. Gill, A.J.; Kolson, D.L. Chronic inflammation and the role for cofactors (hepatitis C, drug abuse, antiretroviral drug toxicity, aging) in HAND persistence. Curr. HIV/AIDS Rep. 2014, 11, 325-335. [CrossRef] [PubMed]

69. Gill, A.J.; Garza, R.; Ambegaokar, S.S.; Gelman, B.B.; Kolson, D.L. Heme oxygenase-1 promoter region (GT)n polymorphism associates with increased neuroimmune activation and risk for encephalitis in HIV infection. J. Neuroinflamm. 2018, 15, 70. [CrossRef] [PubMed]

70. Mollace, V.; Nottet, H.S.; Clayette, P.; Turco, M.C.; Muscoli, C.; Salvemini, D.; Perno, C.F. Oxidative stress and neuroAIDS: Triggers, modulators and novel antioxidants. Trends Neurosci. 2001, 24, 411-416. [CrossRef]

71. Hulgan, T.; Morrow, J.; D’Aquila, R.T.; Raffanti, S.; Morgan, M.; Rebeiro, P.; Haas, D.W. Oxidant stress is increased during treatment of human immunodeficiency virus infection. Clin. Infect. Dis. 2003, 37, 1711-1717. [CrossRef]

72. Suneetha, A.; Raja, R.K. Comparison of LC-UV and LC-MS methods for simultaneous determination of teriflunomide, dimethyl fumarate and fampridine in human plasma: Application to rat pharmacokinetic study. Biomed. Chromatogr. 2016, 30, 1371-1377. [CrossRef]

73. Gold, R.; Linker, R.A.; Stangel, M. Fumaric acid and its esters: An emerging treatment for multiple sclerosis with antioxidative mechanism of action. Clin. Immunol. 2012, 142, 44-48. [CrossRef]

74. Kumar, P.; Sharma, G.; Kumar, R.; Malik, R.; Singh, B.; Katare, O.P.; Raza, K. Enhanced Brain Delivery of Dimethyl Fumarate Employing Tocopherol-Acetate-Based Nanolipidic Carriers: Evidence from Pharmacokinetic, Biodistribution, and Cellular Uptake Studies. ACS Chem. Neurosci. 2017, 8, 860-865. [CrossRef]

75. Beaver, S.K.; Mesa-Torres, N.; Pey, A.L.; Timson, D.J. NQO1: A target for the treatment of cancer and neurological diseases, and a model to understand loss of function disease mechanisms. Biochim. Biophys. Acta Proteins Proteom. 2019, 1867, 663-676. [CrossRef]

76. Starkov, A.A.; Andreyev, A.Y.; Zhang, S.F.; Starkova, N.N.; Korneeva, M.; Syromyatnikov, M.; Popov, V.N. Scavenging of H2O2 by mouse brain mitochondria. J. Bioenerg. Biomembr. 2014, 46, 471-477. [CrossRef]

77. Jin, L.; Li, D.; Alesi, G.N.; Fan, J.; Kang, H.B.; Lu, Z.; Boggon, T.J.; Jin, P.; Yi, H.; Wright, E.R.; et al. Glutamate dehydrogenase 1 signals through antioxidant glutathione peroxidase 1 to regulate redox homeostasis and tumor growth. Cancer Cell 2015, 27, 257-270. [CrossRef]

78. Basuroy, S.; Bhattacharya, S.; Tcheranova, D.; Qu, Y.; Regan, R.F.; Leffler, C.W.; Parfenova, H. HO-2 provides endogenous protection against oxidative stress and apoptosis caused by TNF-alpha in cerebral vascular endothelial cells. Am. J. Physiol. Cell Physiol. 2006, 291, C897-C908. [CrossRef]

79. Chang, E.F.; Wong, R.J.; Vreman, H.J.; Igarashi, T.; Galo, E.; Sharp, F.R.; Stevenson, D.K.; Noble-Haeusslein, L.J. Heme oxygenase-2 protects against lipid peroxidation-mediated cell loss and impaired motor recovery after traumatic brain injury. J. Neurosci. 2003, 23, 3689-3696. [CrossRef]

80. Chen, J. Heme oxygenase in neuroprotection: From mechanisms to therapeutic implications. Rev. Neurosci. 2014, 25, 269-280. [CrossRef]

81. Chen, J.; Tu, Y.; Connolly, E.C.; Ronnett, G.V. Heme oxygenase-2 protects against glutathione depletion-induced neuronal apoptosis mediated by bilirubin and cyclic GMP. Curr. Neurovasc. Res. 2005, 2, 121-131. [CrossRef]

82. Goemaere, J.; Knoops, B. Peroxiredoxin distribution in the mouse brain with emphasis on neuronal populations affected in neurodegenerative disorders. J. Comp. Neurol. 2012, 520, 258-280. [CrossRef] 
83. Kim, S.U.; Park, Y.H.; Min, J.S.; Sun, H.N.; Han, Y.H.; Hua, J.M.; Lee, T.H.; Lee, S.R.; Chang, K.T.; Kang, S.W.; et al. Peroxiredoxin $\mathrm{I}$ is a ROS/p38 MAPK-dependent inducible antioxidant that regulates NF-kappaB-mediated iNOS induction and microglial activation. J. Neuroimmunol. 2013, 259, 26-36. [CrossRef]

84. Neumann, C.A.; Cao, J.; Manevich, Y. Peroxiredoxin 1 and its role in cell signaling. Cell Cycle 2009, 8, 4072-4078. [CrossRef]

85. Wallace, D.C. Mitochondrial genetic medicine. Nat. Genet. 2018, 50, 1642-1649. [CrossRef]

86. Krebs, H.A. Rate control of the tricarboxylic acid cycle. Adv. Enzyme Regul. 1970, 8, 335-353. [CrossRef]

87. Chance, B.; Baltscheffsky, H. Respiratory enzymes in oxidative phosphorylation. VII. Binding of intramitochondrial reduced pyridine nucleotide. J. Biol. Chem. 1958, 233, 736-739. [CrossRef]

88. Chance, B.; Cohen, P.; Jobsis, F.; Schoener, B. Intracellular oxidation-reduction states in vivo. Science 1962, 137, 499-508. [CrossRef]

89. Chance, B.; Schoener, B. High and low energy states of cytochromes. I. In mitochondria. J. Biol. Chem. 1966, 241, 4567-4573. [CrossRef]

90. Chance, B.; Ernster, L.; Garland, P.B.; Lee, C.P.; Light, P.A.; Ohnishi, T.; Ragan, C.I.; Wong, D. Flavoproteins of the mitochondrial respiratory chain. Proc. Natl. Acad. Sci. USA 1967, 57, 1498-1505. [CrossRef] [PubMed]

91. Cortassa, S.; O'Rourke, B.; Aon, M.A. Redox-optimized ROS balance and the relationship between mitochondrial respiration and ROS. Biochim. Biophys. Acta 2014, 1837, 287-295. [CrossRef]

92. Zorov, D.B.; Juhaszova, M.; Sollott, S.J. Mitochondrial reactive oxygen species (ROS) and ROS-induced ROS release. Physiol. Rev. 2014, 94, 909-950. [CrossRef]

93. Jones, D.P.; Sies, H. The Redox Code. Antioxid. Redox Signal. 2015, 23, 734-746. [CrossRef] [PubMed]

94. Quinn, K.P.; Sridharan, G.V.; Hayden, R.S.; Kaplan, D.L.; Lee, K.; Georgakoudi, I. Quantitative metabolic imaging using endogenous fluorescence to detect stem cell differentiation. Sci. Rep. 2013, 3, 3432. [CrossRef]

95. Varone, A.; Xylas, J.; Quinn, K.P.; Pouli, D.; Sridharan, G.; McLaughlin-Drubin, M.E.; Alonzo, C.; Lee, K.; Munger, K.; Georgakoudi, I. Endogenous two-photon fluorescence imaging elucidates metabolic changes related to enhanced glycolysis and glutamine consumption in precancerous epithelial tissues. Cancer Res. 2014, 74, 3067-3075. [CrossRef] [PubMed]

96. Podsednik, A.; Jacob, A.; Li, L.Z.; Xu, H.N. Relationship between Optical Redox Status and Reactive Oxygen Species in Cancer Cells. React. Oxyg. Species (Apex) 2020, 9, 95-108. [CrossRef] [PubMed]

97. Lautrup, S.; Sinclair, D.A.; Mattson, M.P.; Fang, E.F. NAD(+) in Brain Aging and Neurodegenerative Disorders. Cell Metab. 2019, 30, 630-655. [CrossRef] [PubMed]

98. Ghosh, D.; LeVault, K.R.; Barnett, A.J.; Brewer, G.J. A reversible early oxidized redox state that precedes macromolecular ROS damage in aging nontransgenic and 3xTg-AD mouse neurons. J. Neurosci. 2012, 32, 5821-5832. [CrossRef]

99. Akay, C.; Cooper, M.; Odeleye, A.; Jensen, B.K.; White, M.G.; Vassoler, F.; Gannon, P.J.; Mankowski, J.; Dorsey, J.L.; Buch, A.M.; et al. Antiretroviral drugs induce oxidative stress and neuronal damage in the central nervous system. J. Neurovirol. 2014, 20, 39-53. [CrossRef]

100. Kolgiri, V.; Nagar, V.; Patil, V. Association of serum total bilirubin and plasma 8-OHdG in HIV/AIDS patients. Interv. Med. Appl. Sci. 2018, 10, 76-82. [CrossRef]

101. Ande, A.; Sinha, N.; Rao, P.S.; McArthur, C.P.; Ayuk, L.; Achu, P.N.; Njinda, A.; Kumar, A.; Kumar, S. Enhanced oxidative stress by alcohol use in HIV+ patients: Possible involvement of cytochrome P450 2E1 and antioxidant enzymes. AIDS Res. Ther. 2015, 12, 29. [CrossRef] [PubMed]

102. Kolgiri, V.; Patil, V.W. Protein carbonyl content: A novel biomarker for aging in HIV/AIDS patients. Braz. J. Infect. Dis. 2017, 21, 35-41. [CrossRef]

103. Botha, J.; Fabian, J.; Etheredge, H.; Conradie, F.; Tiemessen, C.T. HIV and Solid Organ Transplantation: Where Are we Now. Curr. HIV/AIDS Rep. 2019, 16, 404-443. [CrossRef] [PubMed] 